\title{
Active Aeroelastic Wing Aerodynamic Model Development and Validation for a Modified F/A-18A
}

\author{
Stephen B. Cumming* \\ NASA Dryden Flight Research Center, Edwards, CA, 93523 \\ Corey G. Diebler ${ }^{\dagger}$ \\ NASA Kennedy Space Center, Kennedy Space Center, FL, 32899
}

\begin{abstract}
A new aerodynamic model has been developed and validated for a modified F/A-18A used for the Active Aeroelastic Wing (AAW) research program. The goal of the program was to demonstrate the advantages of using the inherent flexibility of an aircraft to enhance its performance. The research aircraft was an F/A-18A with wings modified to reduce stiffness and a new control system to increase control authority. There have been two flight phases. Data gathered from the first flight phase were used to create the new aerodynamic model. A maximum-likelihood output-error parameter estimation technique was used to obtain stability and control derivatives. The derivatives were incorporated into the National Aeronautics and Space Administration F-18 simulation, validated, and used to develop new AAW control laws. The second phase of flights was used to evaluate the handling qualities of the AAW aircraft and the control law design process, and to further test the accuracy of the new model. The flight test envelope covered Mach numbers between 0.85 and 1.30 and dynamic pressures from 600 to 1250 pound-force per square foot. The results presented in this report demonstrate that a thorough parameter identification analysis can be used to improve upon models that were developed using other means. This report describes the parameter estimation technique used, details the validation techniques, discusses differences between previously existing F/A-18 models, and presents results from the second phase of research flights.
\end{abstract}

\section{Nomenclature}

$\begin{array}{ll}\text { AAW } & =\text { Active Aeroelastic Wing } \\ \mathrm{AIL} & =\text { aileron } \\ \mathrm{c} & =\text { mean aerodynamic chord } \\ \mathrm{CG} & =\text { center of gravity } \\ C_{l_{d T E F}} & \text { rolling-moment coefficient due to differential TEF deflection } \\ C_{m} & =\text { pitching-moment coefficient } \\ C_{m_{\alpha}} & =\text { pitching-moment coefficient due to angle of attack } \\ C_{m_{I L E F}} & \text { pitching-moment coefficient due to symmetric ILEF deflection } \\ C_{m_{q}} & =\text { pitching-moment coefficient due to dimensionless pitch rate } \\ C_{n_{d T E F}} & =\text { yawing-moment coefficient due to differential TEF deflection } \\ C_{N_{\alpha}} & =\text { normal-force coefficient due to angle of attack } \\ \mathrm{d} & =\text { differential surface deflection } \\ g & =\text { acceleration due to gravity } \\ \text { ILEF } & =\text { inboard leading-edge flap } \\ \text { ITB } & =\text { integrated test block } \\ \text { LEF } & =\text { leading-edge flap }\end{array}$

\footnotetext{
* Aerospace Engineer, Aerodynamics Branch, P.O. Box 273/Mail Stop D-2228, AIAA Member.

${ }^{\dagger}$ Safety Engineer, Shuttle Safety \& Mission Assurance, John F. Kennedy Space Center/Mail Stop SA-B1.
} 


$\begin{array}{ll}\text { NASA } & =\text { National Aeronautics and Space Administration } \\ \text { OBES } & =\text { Onboard Excitation System } \\ \text { OLEF } & =\text { outboard leading-edge flap } \\ \text { PID } & =\text { parameter identification } \\ \text { POPU } & =\text { push-over-pull-up } \\ \text { q } & =\text { pitch rate } \\ \text { RFCS } & =\text { Research Flight Control System } \\ \text { RPO } & =\text { rolling pull-out } \\ \text { RUD } & =\text { rudder } \\ \text { STAB } & =\text { stabilator } \\ \text { SRA } & =\text { Systems Research Aircraft } \\ \text { TEF } & =\text { trailing edge flap } \\ \text { V } & =\text { true airspeed } \\ \alpha, \text { alpha } & =\text { angle of attack } \\ \delta, \text { delta } & =\text { control surface deflection }\end{array}$

\section{Introduction}

TN April of 2005, the National Aeronautics and Space Administration (NASA) Dryden Flight Research Center 1 completed flight tests for the Active Aeroelastic Wing (AAW) program. The program was a joint research effort involving NASA, the Air Force Research Lab (AFRL), the United States Navy, and industry. The program looked to develop the multidisciplinary technologies needed to integrate aircraft aerodynamics, active controls, and structural aeroelastic behavior to maximize vehicle performance. ${ }^{1}$ Control laws were designed for a light, flexible-winged aircraft that would improve roll performance by exploiting the elastic characteristics of the wing. ${ }^{2}$

At the outset of the flight program, The Boeing Company (Chicago, Illinois) modified a NASA F/A-18A in preparation for flight testing. ${ }^{1}$ In order to develop the integrated control laws, it was necessary that the designers possess an accurate representation of the stability and control characteristics of the aircraft. To acquire this representation, a parameter identification (PID) flight phase was flown to extract the necessary data from flight measurements. This approach had not been pursued during the development of existing F/A-18 aerodynamic models ${ }^{1}$ and resulted in a more accurate model of the aircraft than had previously existed.

This report describes the approach taken to develop and validate the AAW aerodynamic model as well as an informal verification from flight test results. Findings and details about the PID results and model differences are discussed in addition to data from flight tests.

\section{Vehicle Selection}

A NASA Dryden F/A-18A, tail number 853, shown in Fig. 1, was selected as the AAW aircraft for several reasons. First, NASA already had a full F-18 simulation and the team already had a wealth of knowledge about the aircraft and its systems. Second, the leading-edge flap (LEF) of the F/A-18 is split into an inboard and an outboard. A standard F/A-18 LEF is operated as one unit driven by a single actuator so that both sections have the same motion. Additional hardware was added to the AAW aircraft that allowed the inboard section of the LEF to operate independently of the outboard section. This provided an additional set of surfaces with which to control the twist, aerodynamics, and load distribution of the wing. Third, the F/A-18 was chosen as the test aircraft because during preproduction performance testing it was discovered that the lighter preproduction wings were too flexible and did not meet the desired roll performance criteria. For this reason, the F/A-18 was redesigned with a stronger, heavier wing. This helped to improve the F/A-18 roll performance, but it resulted in a heavier aircraft, reducing range and carrying capability. Since the goal of the AAW technology was to improve the roll performance of a flexible wing, the preproduction F/A-18 provided a history in which wing flexibility was detrimental to aircraft performance. 


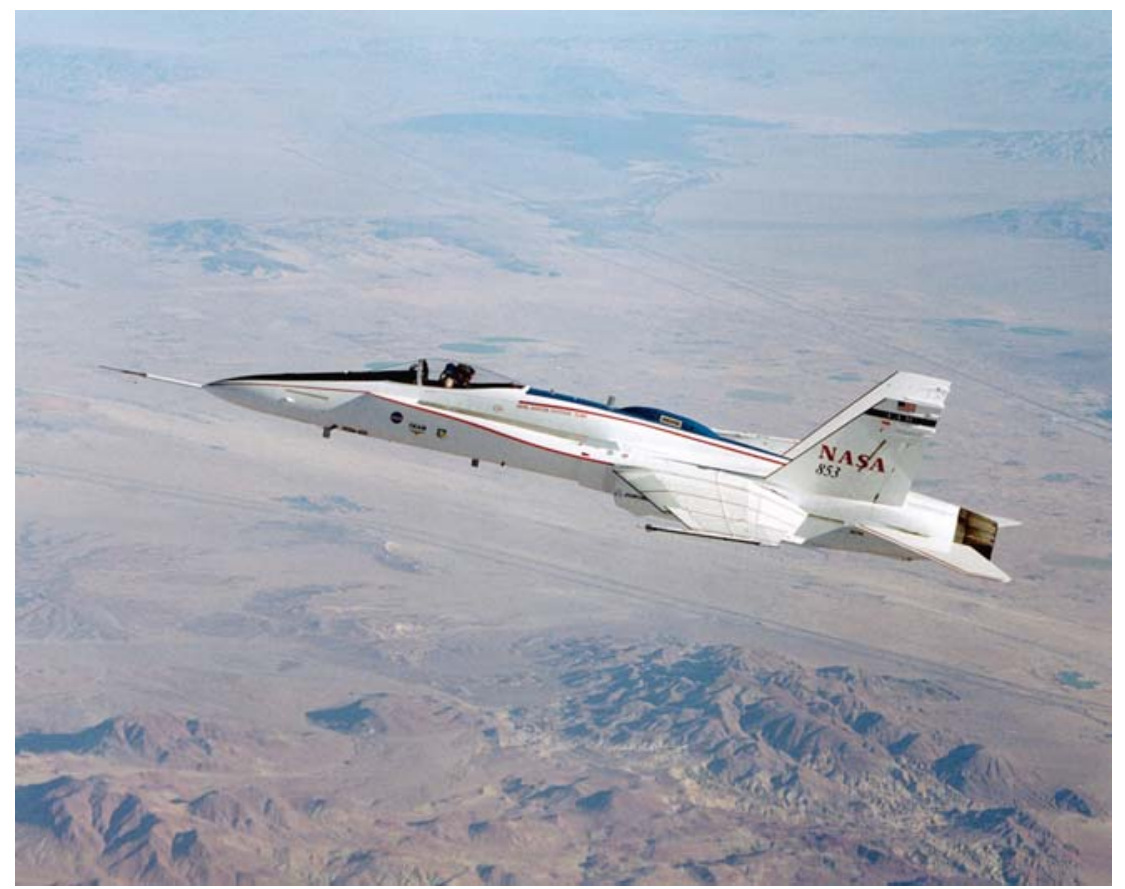

Figure 1. The Active Aeroelastic Wing research aircraft.

\section{Vehicle Modifications}

Several modifications were made to the aircraft for the AAW program. ${ }^{1}$ The wings of the AAW aircraft were returned to the reduced stiffness levels of the preproduction F/A-18 design to provide greater flexibility for flight testing. ${ }^{1}$ The LEF system was also modified. The F/A-18 LEF system, which operates as a single unit, was split at the wing fold into an inboard LEF (ILEF) and outboard LEF (OLEF) that can operate independent of one another. A schematic illustrating the control surfaces can be seen in Fig. 2. The LEF drive system was also modified to give the LEFs a greater range of motion as can be seen in Table 1.

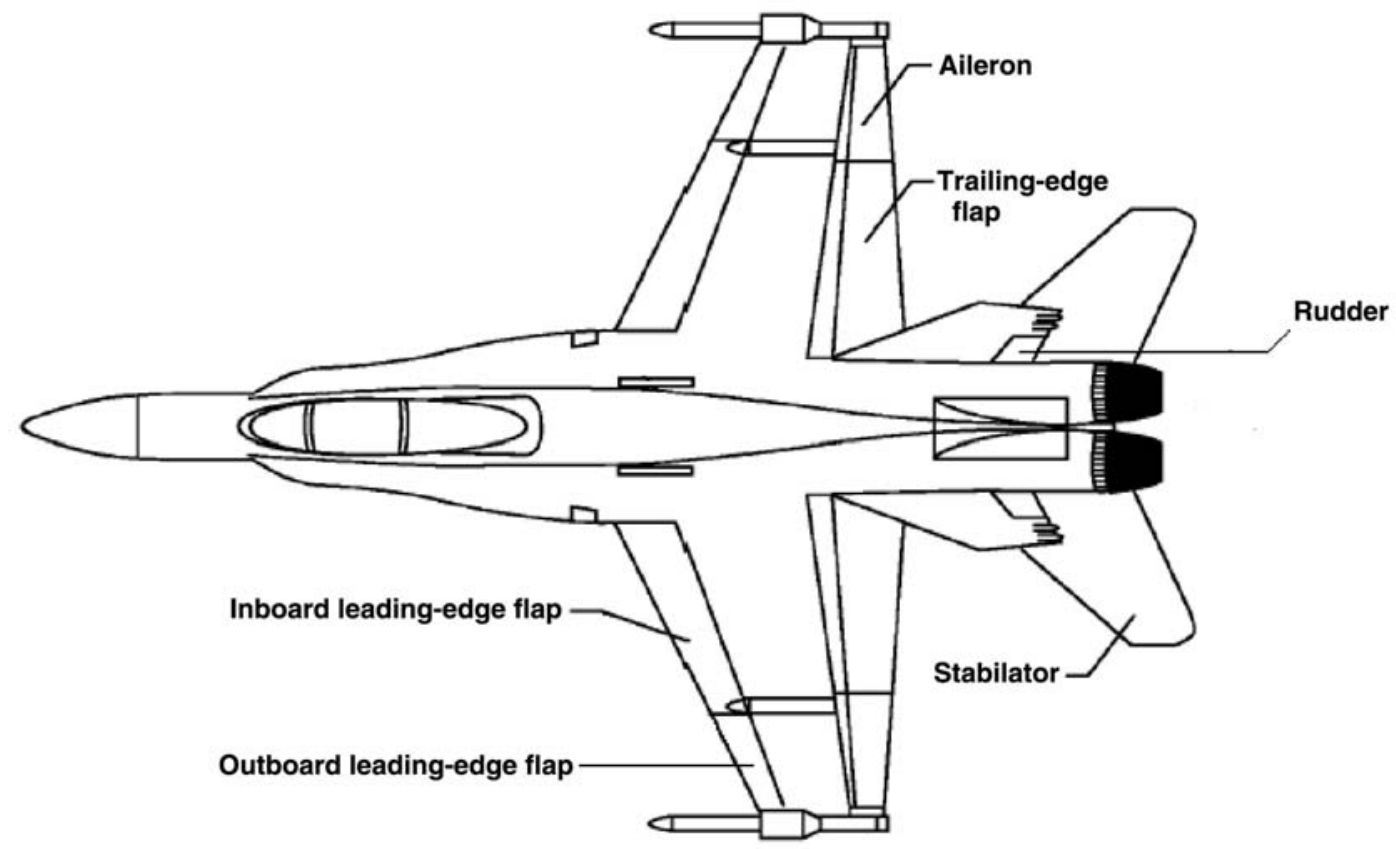

Figure 2. Active Aeroelastic Wing schematic. 
Table 1. Control surface rates and limits.

\begin{tabular}{lccc}
\hline \hline \multicolumn{1}{c}{ Control surface } & $\begin{array}{c}\text { Rate, } \\
\mathrm{deg} / \mathrm{sec}\end{array}$ & $\begin{array}{c}\text { Upward travel, } \\
\mathrm{deg}\end{array}$ & $\begin{array}{c}\text { Downward travel, } \\
\mathrm{deg}\end{array}$ \\
\hline Aileron & 100 & 25 & 45 \\
Inboard leading-edge flap & 15 & 3 & 34 \\
Trailing-edge flaps & 18 & 18 & 45 \\
Stabilator & 40 & 10.5 & 24 \\
Rudder & 56 & 30 (left) & 30 (right) \\
Baseline outboard leading-edge flap & 15 & 3 & 34 \\
$\begin{array}{l}\text { Research flight control system } \\
\text { outboard leading-edge flap }\end{array}$ & 45 & 10 & 34 \\
\hline \hline
\end{tabular}

The AAW flight control computers were modified as well. The standard 701E processor running the basic F/A-18 flight control laws was augmented with a Research Flight Control System (RFCS). The RFCS was added to provide a flexible means to run the research software load when desired and revert back to the standard F/A-18 control laws when needed. The pilot could switch between the standard control laws and the research control laws while in flight by means of a switch in the cockpit. The standard controls were flown during take-off, landing, and while transitioning between test points.

The AAW aircraft was equipped with a complete set of air data, inertial, and control-surface position instrumentation. In addition to the F/A-18 standard instrumentation system, a myriad of sensors were added to the AAW aircraft to meet research objectives. ${ }^{1}$ Data measurements were telemetered to the ground control room for real-time monitoring and were also recorded for postflight analysis.

The analysis discussed in this report used measurements from the standard F/A-18 instrumentation package (obtained through the 1553 data bus) and additional instruments mounted throughout the aircraft which were telemetered down on a different data stream. Ambient temperature, control surface positions, and pitch and roll attitudes were obtained from the standard F/A-18 instrumentation package. A research instrumentation package provided air data measurements (pitot and static pressures, angle of attack, and angle of sideslip) from a noseboom mounted to the airplane, and angular rates and aircraft accelerations from rate gyros and accelerometers. Also, a camera pod was mounted on the top of the aircraft to record the amount of wing twist during maneuvering.

\section{Flight Tests}

In order to ensure a safe, thorough evaluation, the flight research program was planned in two phases. The first phase of AAW flights was dedicated to flutter clearance, loads clearance, and model development (PID maneuvers). The data gathered from these flights were used to create new aerodynamic and loads models to be used in the control law design process. The second phase of flights was dedicated to demonstrating the AAW technology and testing the new flight control laws. Data from the second phase of flights were also used to further validate, or informally verify, the new models.

The AAW test matrix was made up of 18 separate test points: nine subsonic (Mach 0.85, 0.9, and 0.95) and nine supersonic (Mach 1.1, 1.2, and 1.3). Dynamic pressures ranged from 600 to 1500 psf. Figure 3 displays the target AAW test matrix and flight envelope. The pilot flew to the conditions displayed to him by the F/A-18 production air data system. It was found that the production system did not have a good calibration above Mach 1. More accurate data from the research noseboom were used for data analysis, but were not displayed to the pilot in the cockpit. Therefore, the true flight conditions did not exactly match those shown in Fig. 3. 


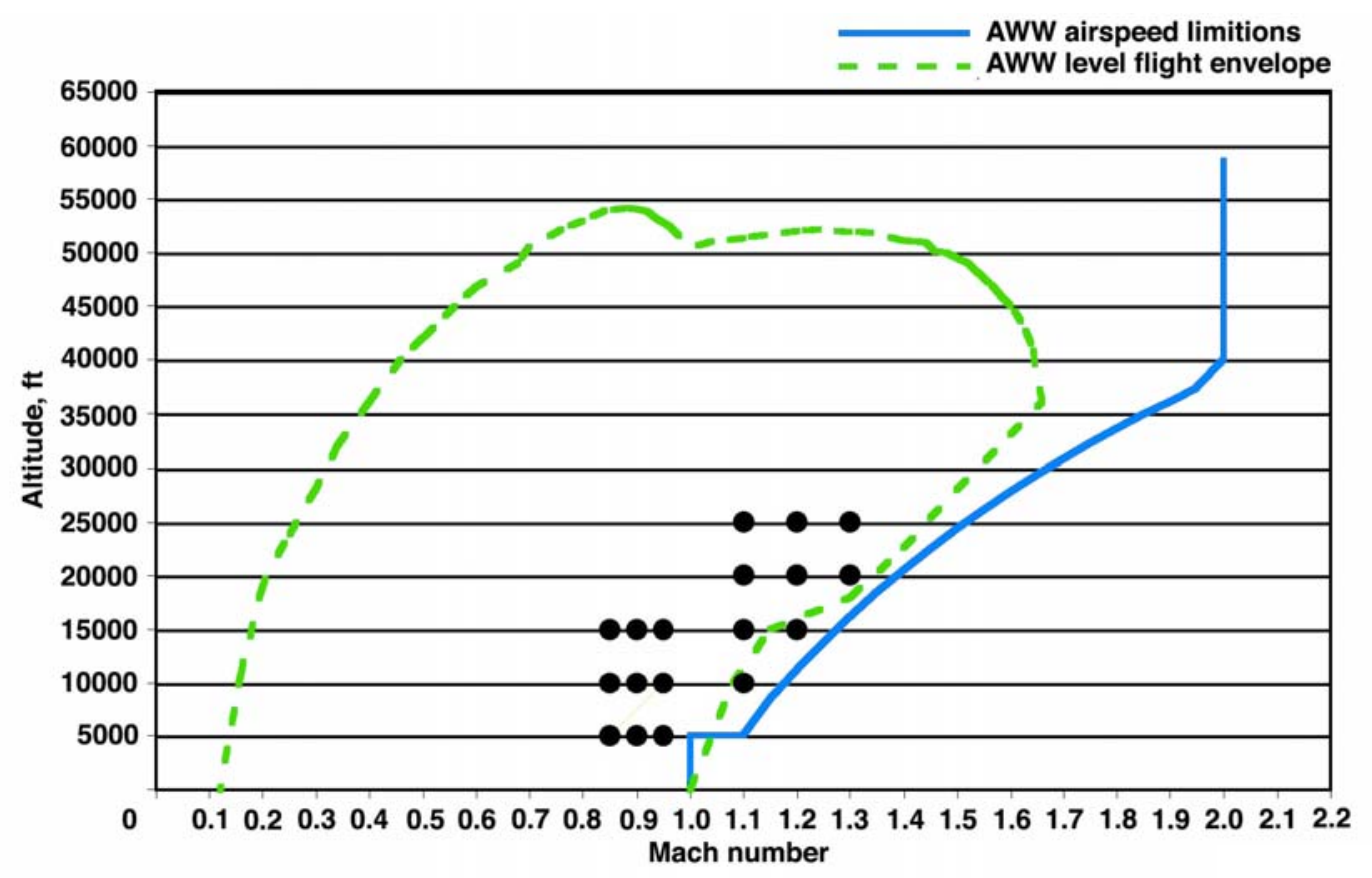

Figure 3. Active Aeroelastic Wing test matrix.

\section{Phase I Parameter Identification}

A total of 51 flights were flown during phase I. Flights consisted of functional checkout, clearance, air data calibration, PID, and Integrated Test Block (ITB) maneuvers.

\section{A. Onboard Excitation System Maneuvers}

During the first phase flights, the RFCS was used to operate the Onboard Excitation System (OBES). The OBES consisted of 31 preprogrammed maneuvers which were added to the underlying 701E commands. The RFCS did not deactivate the standard control laws nor did it eliminate the feedback responses to aircraft rates. This method allowed the 701E to retain control of the aircraft while the OBES maneuvers were flown. Six of the 31 OBES maneuvers were designed as PID maneuvers. The other 25 OBES maneuvers consisted of frequency sweeps to investigate aeroservoelastic effects, LEF ground test maneuvers and OLEF failure emulations.

Standard F/A-18 control laws use multiple surface inputs in response to pilot stick deflections to coordinate the motion of the vehicle. The control surface motion is highly correlated and it is not possible to separate out individual effects. In order to extract accurate stability and control derivatives, it is necessary to have independent surface motion during the maneuvers. The six OBES PID maneuvers commanded longitudinal and lateral-directional control surface doublets independent of any piloted stick motion. Each set of control surfaces was actuated one at a time. This separate actuation provided control surface inputs that were largely independent of one another. Since the underlying F/A-18 control laws were still active during these maneuvers, small amounts of control system feedbacks were present, but surface motion was sufficiently decoupled for PID analysis. A combined inboard and outboard LEF doublet was also programmed into the OBES commands. This doublet was used to verify superposition of combined LEF motion and was not used as part of the PID analysis. Tables 2 and 3 show the deflection magnitudes for each of the OBES PID maneuvers. The columns of the tables are in order of sequence, from left to right. Surface doublets were separated by two seconds to allow the aircraft motion to dampen out. Figures 4 and 5 display sample control surface deflections recorded during longitudinal and lateral-directional OBES maneuvers, respectively. 
Table 2. Longitudinal Onboard Excitation System maneuvers.

\begin{tabular}{lcccccc}
\hline \hline & $\delta_{\mathrm{OLEF}}$, deg & $\delta_{\mathrm{ILEF}}$, deg & $\delta_{\mathrm{TEF}}$, deg & $\delta_{\mathrm{LEF}}$, deg & $\delta_{\mathrm{AIL}}$, deg & $\delta_{\mathrm{STAB}}$, deg \\
\hline Large & 3 & 3 & 5 & 3 & 6 & 0.8 \\
Medium & 2 & 2 & 4 & 2 & 5 & 0.8 \\
Small & 1 & 1 & 3 & 1 & 4 & 0.8 \\
\hline \hline
\end{tabular}

Table 3. Lateral-directional Onboard Excitation System maneuvers.

\begin{tabular}{lccccccc}
\hline \hline & $\delta_{\text {RUD }}$, deg & $\delta_{\text {dOLEF }}$, deg & $\delta_{\text {dILEF }}$, deg & $\delta_{\text {dTEF }}$, deg & $\delta_{\text {dAIL }}$, deg & $\delta_{\text {dSTAB }}$, deg & $\delta_{\text {dLEF }}$, deg \\
\hline Large & 4 & 6 & 6 & 10 & 12 & 6 & 6 \\
Medium & 4 & 4 & 4 & 8 & 10 & 6 & 4 \\
Small & 4 & 2 & 2 & 6 & 8 & 2 \\
\hline \hline
\end{tabular}

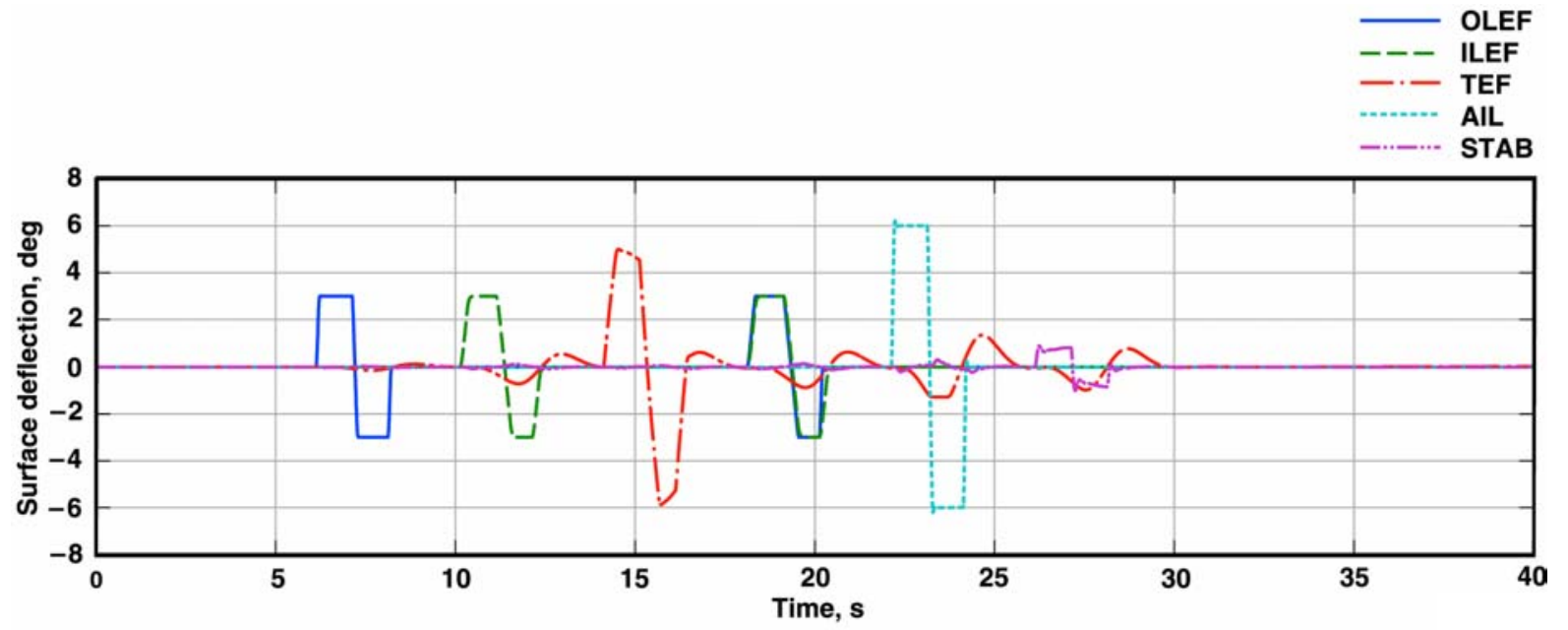

Figure 4. Longitudinal Onboard Excitation System maneuver.

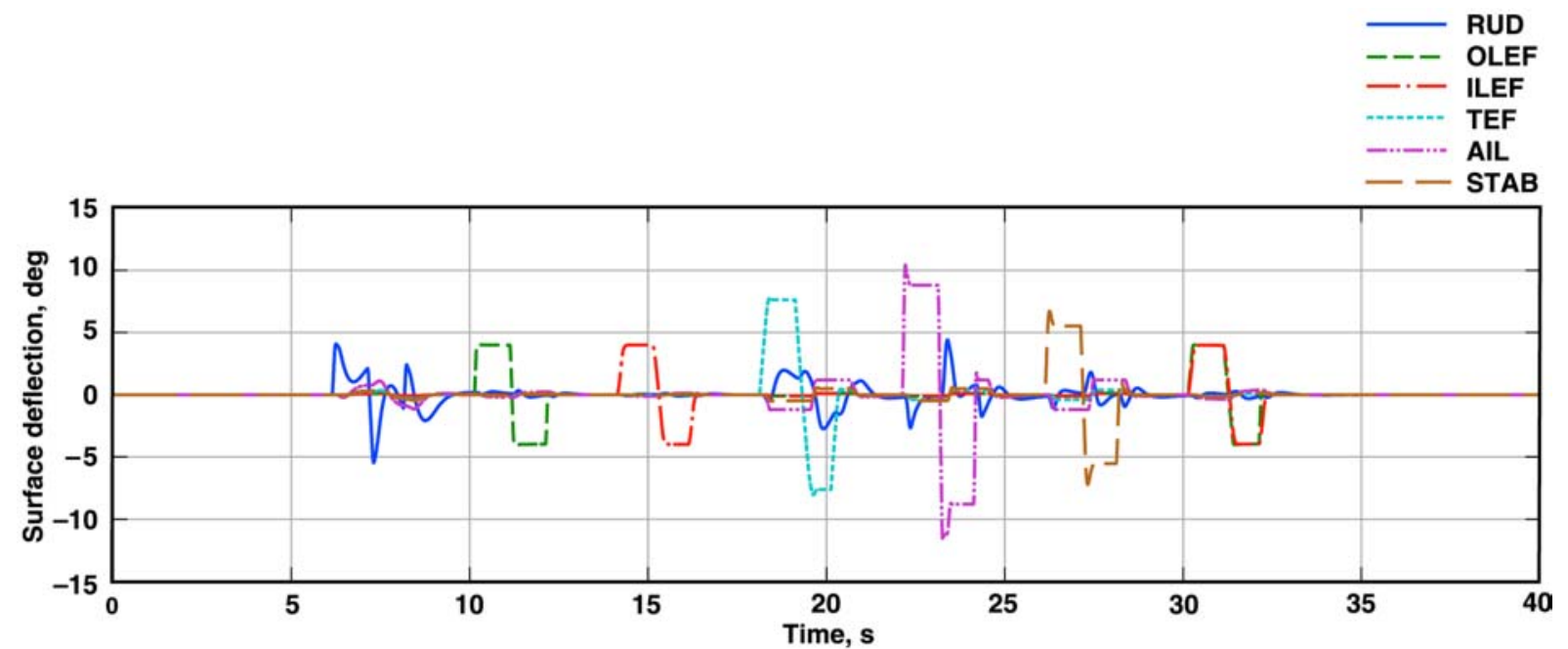

Figure 5. Lateral-directional Onboard Excitation System maneuver. 
Control surface deflections were defined as follows:

Symmetric deflections:

$$
\begin{aligned}
\delta_{\mathrm{AIL}} & =0.5\left(\delta_{\mathrm{AIL}_{\text {Left }}}+\delta_{\mathrm{AIL}_{\text {Right }}}\right) \\
\delta_{\mathrm{STAB}} & =0.5\left(\delta_{\mathrm{STAB}_{\text {Left }}}+\delta_{\mathrm{STAB}_{\text {Right }}}\right) \\
\delta_{\mathrm{TEF}} & =0.5\left(\delta_{\mathrm{TEF}_{\text {Left }}}+\delta_{\left.\mathrm{TEF}_{\text {Right }}\right)}\right) \\
\delta_{\mathrm{ILEF}} & =0.5\left(\delta_{\mathrm{ILEF}_{\text {Left }}}+\delta_{\mathrm{ILEF}_{\text {Right }}}\right) \\
\delta_{\mathrm{OLEF}} & =0.5\left(\delta_{\mathrm{OLEF}_{\text {Left }}}+\delta_{\mathrm{OLEF}_{\text {Right }}}\right) \\
\delta_{\mathrm{RUD}} & =0.5\left(\delta_{\mathrm{RUD}_{\text {Left }}}+\delta_{\mathrm{RUD}_{\text {Right }}}\right)
\end{aligned}
$$

Differential deflections:

$$
\begin{aligned}
\delta_{\mathrm{dAIL}} & =\delta_{\mathrm{AIL}_{\text {Left }}}-\delta_{\mathrm{AIL}_{\text {Right }}} \\
\delta_{\mathrm{dSTAB}} & =\delta_{\mathrm{STAB}_{\text {Left }}}-\delta_{\mathrm{STAB}_{\text {Right }}} \\
\delta_{\mathrm{dTEF}} & =\delta_{\mathrm{TEF}_{\text {Left }}}-\delta_{\mathrm{TEF}_{\text {Right }}} \\
\delta_{\mathrm{dILEF}} & =\delta_{\mathrm{ILEF}_{\text {Left }}}-\delta_{\mathrm{ILEF}_{\text {Right }}} \\
\delta_{\mathrm{dOLEF}} & =\delta_{\mathrm{OLEF}_{\text {Left }}}-\delta_{\mathrm{OLEF}_{\text {Right }}}
\end{aligned}
$$

\section{B. Integrated Test Block Maneuvers}

In addition to the OBES maneuvers, ITB maneuvers were flown at each test point. The maneuvers consisted of push-over-pull-ups (POPU), stick rolls, 4- $g$ rolling pull-outs (RPO), and wind-up turns to $5 g$ 's. The $g$ limits were imposed to give a wide range of data, but stay within safe limits. The pilot flew POPUs by pushing forward on the stick to $-1 g$ and then pulling back to $3 g$ 's. The RPOs were flown by banking the airplane, pulling back on the stick to maintain a level 4-g turn, then quickly rolling the airplane $180 \mathrm{deg}$ in the opposite direction while maintaining constant longitudinal stick position. The wind-up turns consisted of the pilot banking the aircraft and pulling back on the stick, slowly increasing the normal acceleration to $5 \mathrm{~g}$ 's while maintaining a constant altitude. These maneuvers were flown using the standard F/A-18 control laws and were used to validate the new aerodynamic model.

\section{PID Technique}

A maximum-likelihood output-error PID technique was used to estimate the stability and control derivatives of the AAW aircraft at each test point. Measured aircraft responses to the predefined OBES maneuvers were read into a PID program known as pEst. ${ }^{3}$ The pEst program used a mathematical cost function to quantify the difference between flight-measured aircraft responses and the responses predicted by a vector set of dynamic equations of motion. ${ }^{4}$ The program then used an iterative technique in which the unknown stability and control parameter values were adjusted to reduce the value of the cost function and thereby increase the agreement of the math model to flight data. This technique resulted in a set of stability and control parameter values for each maneuver analyzed. Figure 6 shows a flow diagram of the pEst process. Parameter values from multiple maneuvers at the same test point were averaged to obtain final values that were then used to create the new AAW aerodynamic model. Figure 7 displays 
the fit from a sample pEst case. Blue lines display flight data, red lines display the predicted math model responses using the final parameter values for this particular run. Because of data sensitivity, all plots in this report showing flight data have the scaling removed.

In order to extract accurate stability and control parameters from the flight data, it was necessary to have precise knowledge of the weight, center of gravity (CG), and rotational moments of inertia, for each maneuver of the aircraft. Thus, a weight-and-balance ground test was performed to measure the unfueled weight and the CG location of the AAW aircraft. Capacitance sensors installed in each fuel tank provided fuel quantities during each maneuver. Fuel data was used postflight along with the tank location to adjust the CG location and inertias.

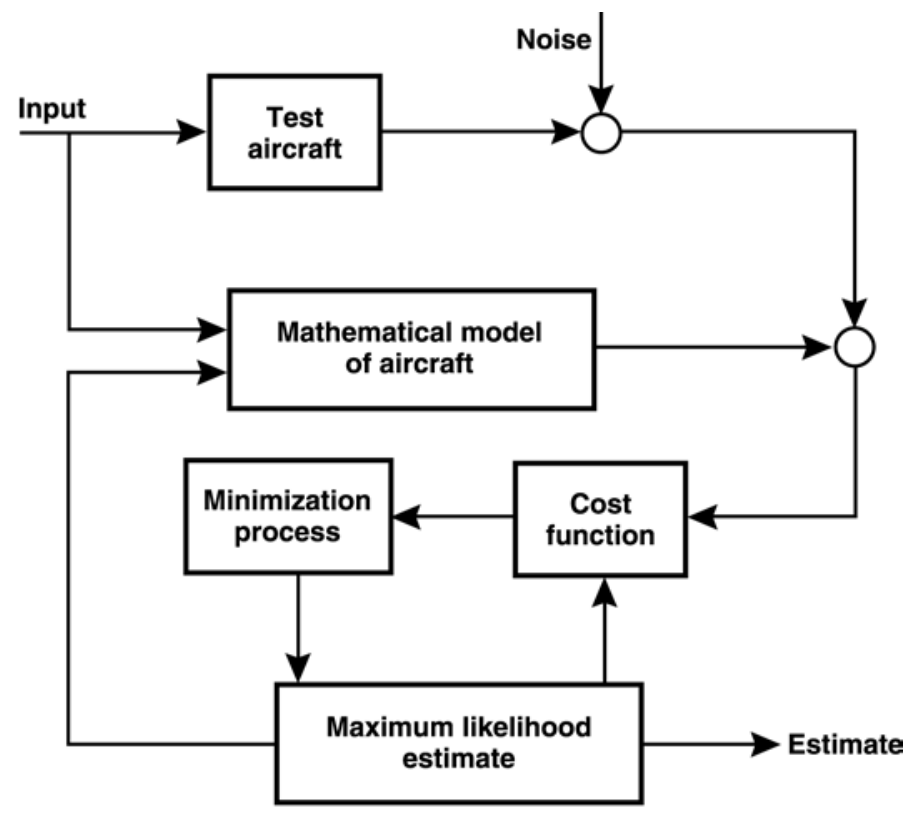

Figure 6. The output-error maximum-likelihood parameter identification technique. 

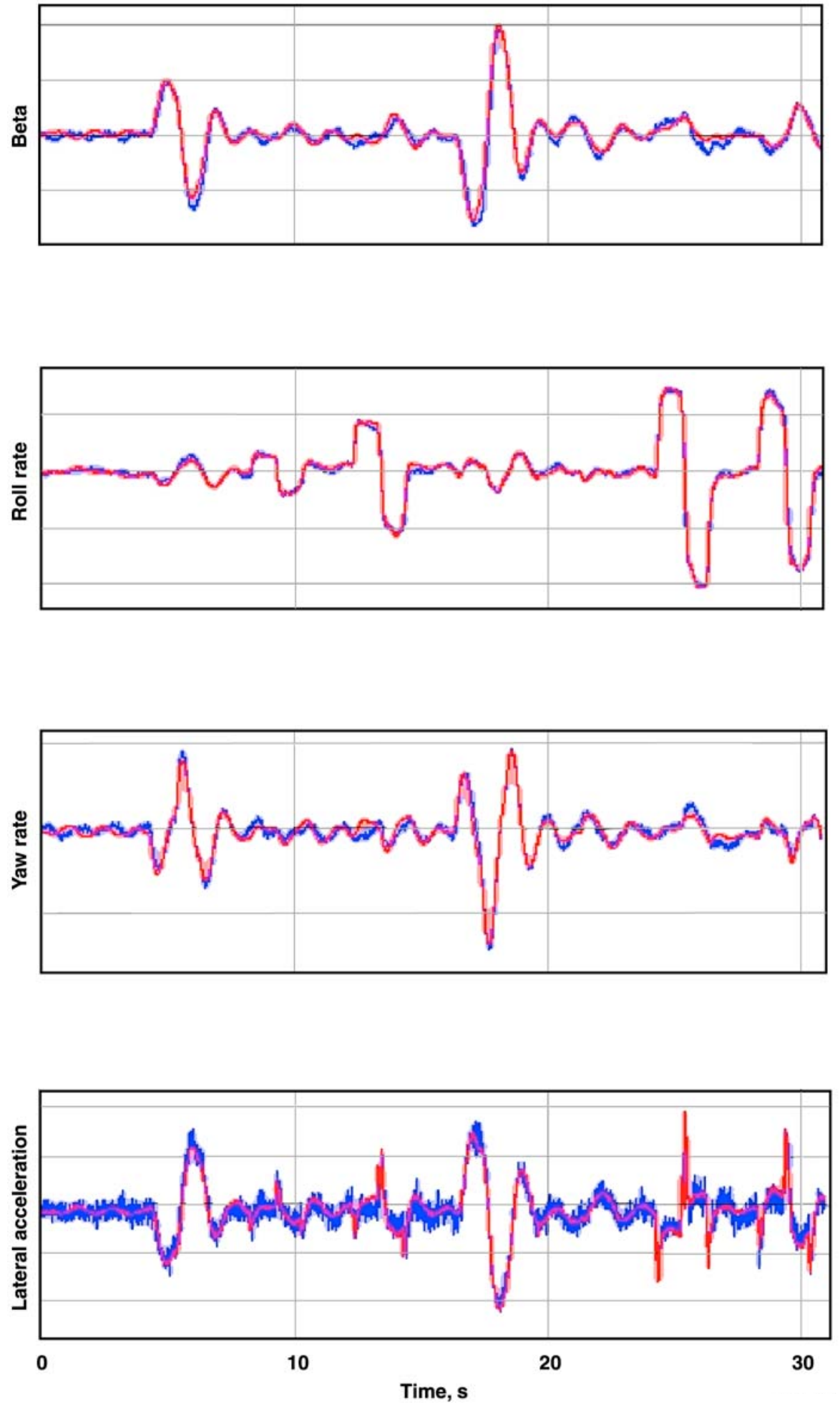

Figure 7. Sample pEst comparison, lateral-directional Onboard Excitation System maneuver at low-altitude, supersonic conditions. 


\section{PID Analysis and Results}

Initially, only OBES maneuvers were used to create the new aerodynamic model. However, during model development, it was discovered that the linear results produced by the pEst analysis were not able to produce the normal accelerations seen during wind-up turns. One possible explanation is that the longitudinal OBES maneuvers alone did not produce a wide enough data range to extract $C_{N_{\alpha}}$ values that could be extrapolated to higher alphas. To alleviate this problem, wind-up turns and POPUs were used in conjunction with the longitudinal OBES maneuvers in order to widen the angle-of-attack and normal acceleration ranges used in the analysis. First, the OBES maneuvers were analyzed to obtain the complete set of stability and control derivatives. Then, while keeping the control derivatives fixed, a POPU or wind-up turn was analyzed to adjust $C_{N_{\alpha}}$. While this approach slightly compromised the match to the OBES data, it worked very well to eliminate the discrepancies seen in high- $g$ maneuvers. Figures 8 and 9, respectively, illustrate the differences between the first version of the model, created using only the OBES maneuvers, and the second version of the model, which utilized wind-up turns and POPUs along with the OBES maneuvers in its creation.
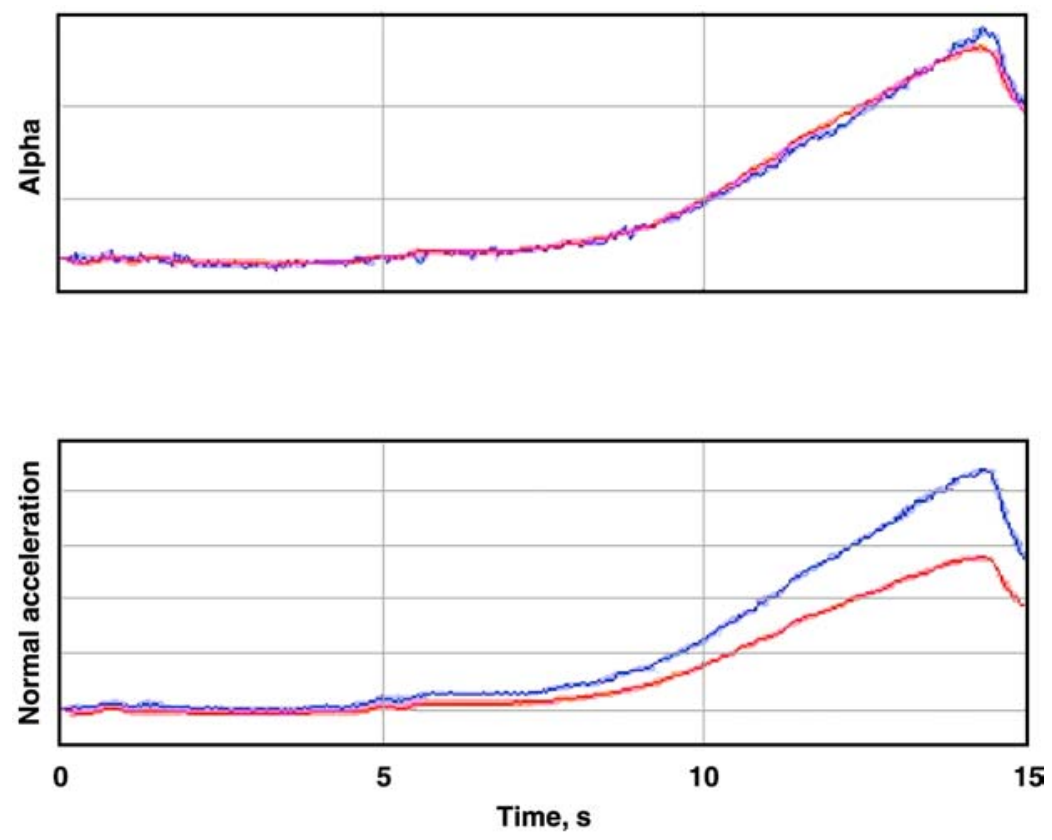

Figure 8. Aerodynamic model version 1.0, wind-up turn at Mach 1.2 and an altitude of 20,000 feet. 

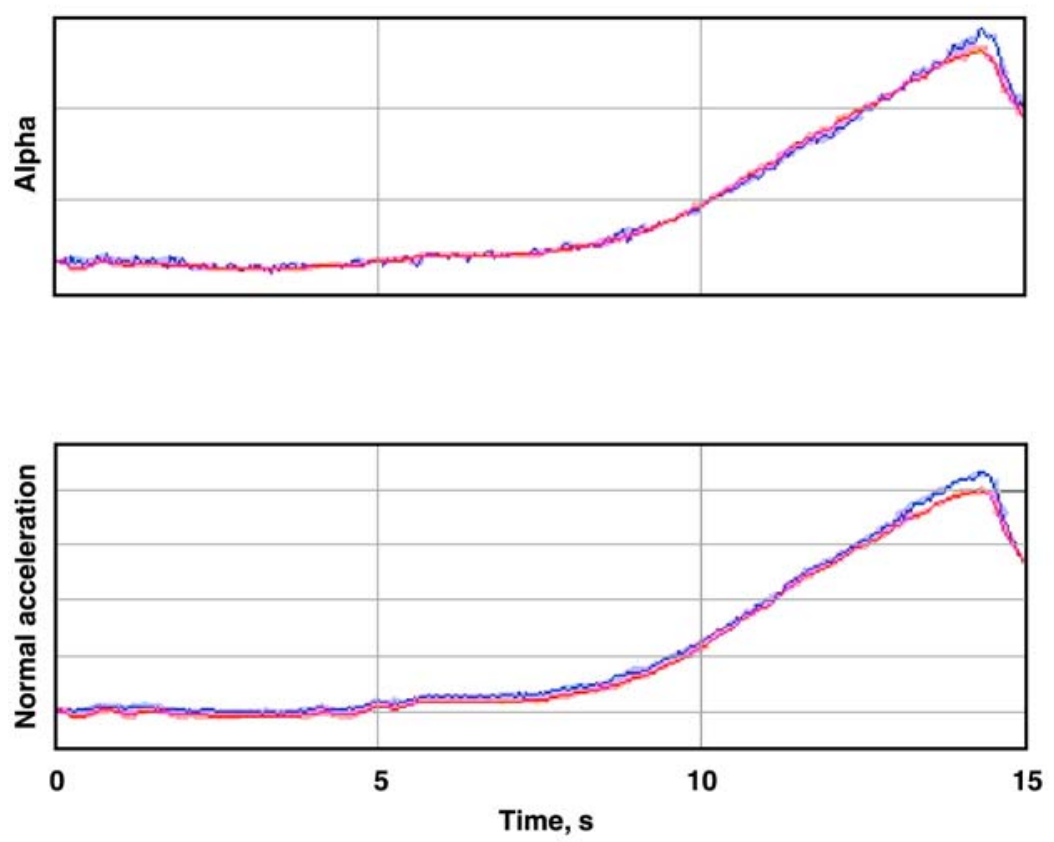

Figure 9. Aerodynamic model version 2.0, wind-up turn at Mach 1.2 and an altitude of 20,000 feet.

Since it was found that the aerodynamic model did not produce valid longitudinal results outside the ranges used in the analysis, limits were imposed on the use of the model. The model was considered valid from 0 to $5 \mathrm{~g}$ 's, roughly the range used during the analysis. A similar investigation was conducted on the lateral-directional results; however, the linearity appeared to hold. Therefore, no limits were imposed on lateral-directional applications.

After the analysis was completed, it was noted that several substantial differences existed between the AAW PID results and existing F/A-18 databases. These findings are consistent with the findings of a previous F/A-18 flight research program conducted at the NASA Dryden Flight Research Center. ${ }^{5}$

In 1999 the NASA Systems Research Aircraft (SRA) completed a series of flight tests that were a precursor to the AAW flights. The SRA aircraft was outfitted with an RFCS and given OBES software nearly identical to that of the AAW aircraft. The major differences between the SRA and the AAW aircraft were that the SRA was an F/A-18B model with unmodified wings and an LEF system that was not divided into inboard and outboard LEFs.

The SRA flew similar OBES PID maneuvers at each of the AAW test points. It was also noted during the SRA program that there were several areas of substantial difference between the flight-derived stability and control parameters and those of the existing F/A-18 models. ${ }^{5}$ The same differences were seen in the AAW results. The most notable of these differences was in the $C_{n_{d T E F}}$ values. Figure 10 shows some of the TEF results at an altitude of $15,000 \mathrm{ft}$. While all models show similar results at all Mach numbers for $C_{l_{d T E F}}$, the same cannot be said for the $C_{n_{\text {dTEF }}}$ values. The AAW and SRA models have $C_{n_{\text {dTEF }}}$ values that remain relatively constant throughout the Mach range while earlier models have $C_{n_{d T E F}}$ values that drop off substantially at supersonic Mach numbers. 

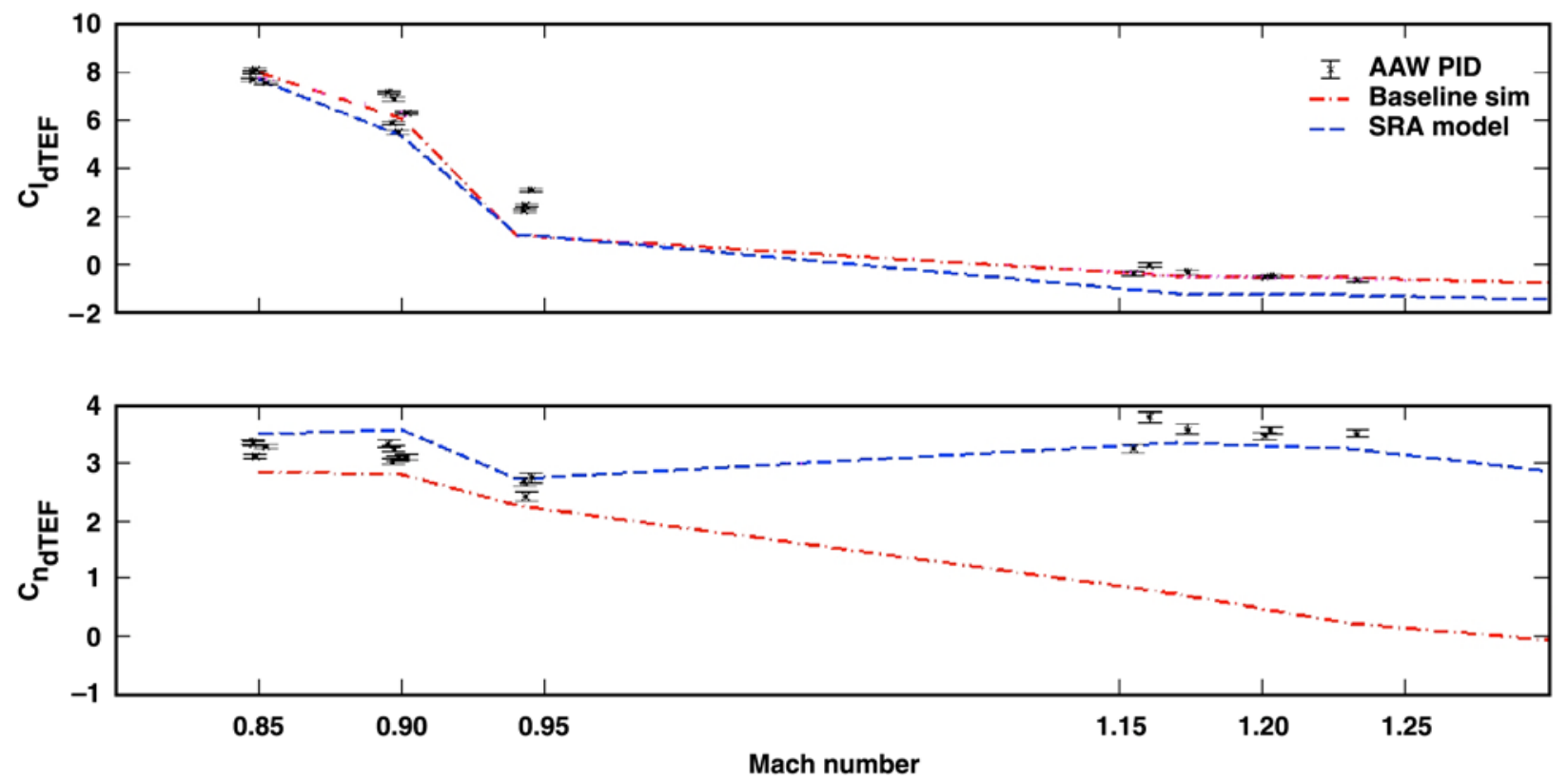

Figure 10. Trailing-edge flap parameter identification results.

During validation it was found that the AAW model provided a much better match to the measured response of a TEF doublet than did the baseline model as can clearly be seen in Fig. 11. This area is one in which the AAW and the SRA models greatly improved upon the standard models. 

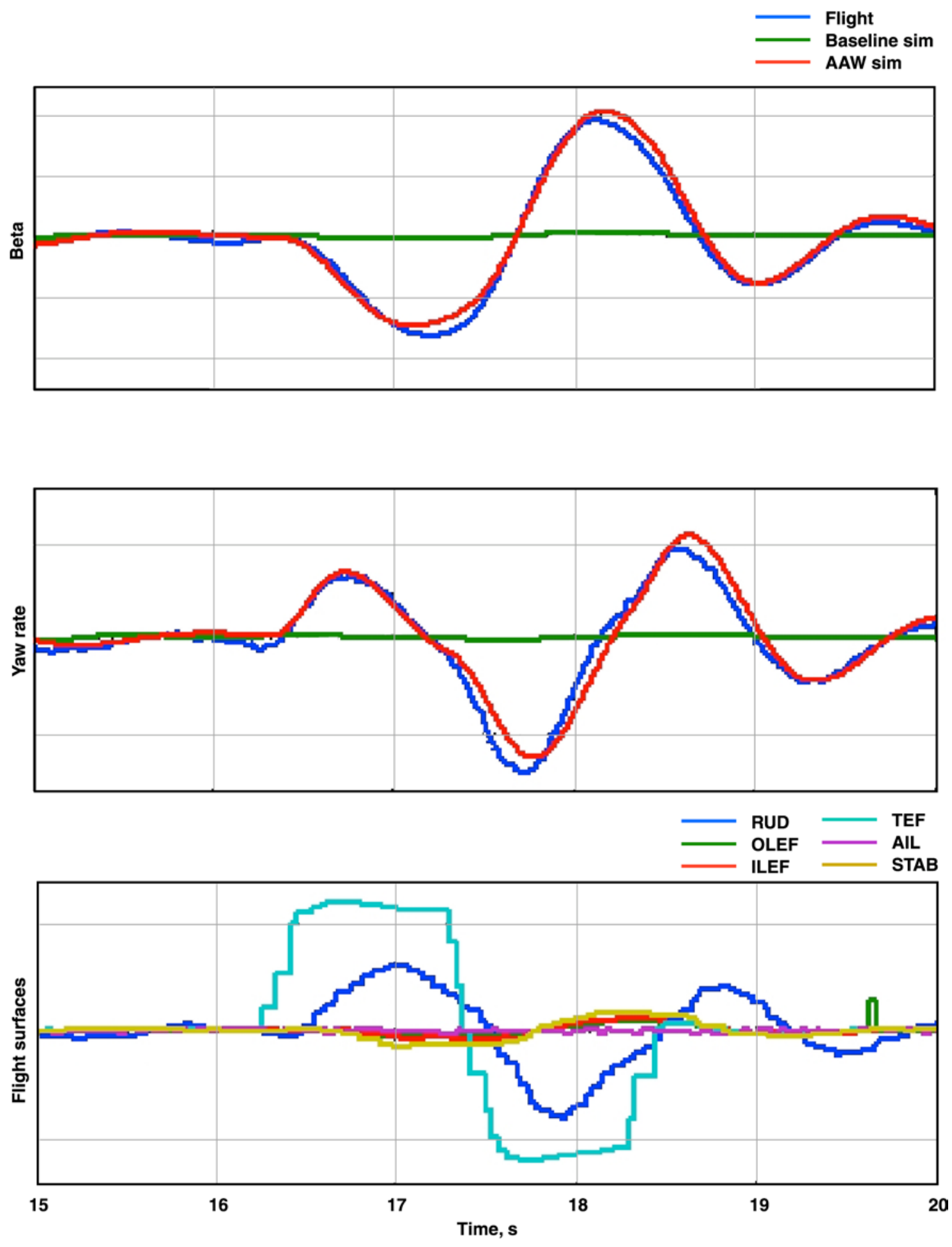

Figure 11. Trailing-edge flap doublet. 


\section{E. Simulation Aerodynamic Model Update}

Once the new aerodynamic model was created, it was used to update the NASA F-18 simulation database. Since the AAW model is only a subset of the much larger F-18 model, it was necessary that simulation revert back to the baseline F-18 database when outside the AAW test matrix. The baseline F-18 model was used as a base and linear deltas were added to the baseline database so that the simulation derivatives were equal to those of the new AAW model. Deltas were ramped to a zero value outside the AAW test matrix to allow for a smooth transition between the two models.

To create the deltas, baseline parameter values were obtained from the simulator at each of the test points and subtracted from the PID results to obtain the delta value as shown in Eq. (1). The delta values were incorporated into a separate database and added to the simulation. The deltas were then added to the simulation force and moment equations as shown in Eq. (2).

$$
\begin{gathered}
\Delta C_{m_{\alpha}}=C_{m_{\alpha}}{ }^{(\mathrm{PID})}-C_{m_{\alpha}} \text { (baseline model) } \\
\Delta C_{m}=\Delta C_{m_{\alpha}} \alpha+\Delta C_{m_{q}} \frac{\mathrm{qc}}{2 \mathrm{~V}}+\Delta C_{m_{I L E F}} \delta_{\mathrm{ILEF}}+\ldots
\end{gathered}
$$

\section{Phase I Aerodynamic Model Validation}

The validity of any aerodynamic model is demonstrated by its ability to replicate flight data. The AAW model underwent a thorough validation process based on that principle before the second phase of flights began. An accurate aerodynamic model was needed for control law design both to increase the chances of achieving program goals and to ensure a safe second phase of flights. Since the new control laws were going to be designed using the newly developed AAW model, any errors in the model would adversely affect the control laws.

Data from the linear model were compared with flight data from the ITB maneuvers. Then, a complete set of OBES maneuvers and ITB maneuvers were run through the nonlinear, six degree-of-freedom simulation at each test point and compared with flight data. Both open-loop and closed-loop simulation runs were conducted. During open-loop test cases, surface positions measured in flight were used to drive the simulation. This method ensured that control surface motion was identical to that seen in flight and provided an isolated aerodynamic model test. For closed-loop cases, the simulation was driven by the piloted stick and rudder commands measured in flight. This method ensured that the piloted input was identical to that of flight; however, the control laws were allowed to freely command the control surfaces in response to simulated aircraft motion. This approach provided a complete end-to-end test of the entire simulation. Figure 12 shows a comparison of the linear model to flight data.

For comparison purposes, baseline aerodynamic model results were obtained using the same method. Figure 13 shows an open-loop comparison for a supersonic lateral-directional OBES maneuver. It is immediately evident that the baseline model produces large oscillations in sideslip angle (beta), yaw rate, and lateral acceleration. This is not, however, an indication that the baseline model is directionally neutrally stable. Instead, the oscillations are a result of the test method. Since the simulation is driven by flight-measured surface positions in open-loop cases, errors in the model responses are not damped out by control feedbacks. The flight-measured surface motion may even continue to drive the oscillations if sufficiently out of phase. The AAW model, on the other hand, shows an excellent agreement with flight data throughout the maneuver. While the open-loop tests provide an excellent isolated test of the AAW aerodynamic model, it is difficult to compare the AAW model to the baseline because of the oscillations in the yaw axis.

Figure 14 shows a closed-loop comparison of the same maneuver. Since the simulation is driven by the measured pilot stick and rudder inputs, the simulation control laws are free to dampen any rates. This gives a much cleaner baseline model response and it is possible to examine the response for each control surface doublet. Again the most notable difference between the two models can be seen during the trailing-edge flap doublet. The baseline model shows no response while the flight data and the AAW model show a definite response in beta, yaw rate, and lateral acceleration. This response was noted as one of the largest improvements throughout the AAW test matrix. Other improvements can be seen in roll rates for the inboard and outboard LEFs and the stabilator.

The OBES maneuvers discussed in the previous paragraphs have shown substantial improvements in several areas over the baseline model. The ITB comparisons further tested the model to see if these improvements would carry over to larger coordinated maneuvers. Figure 15 shows a comparison of a rolling maneuver at the same test point. Again the most obvious differences can be seen in beta and lateral acceleration. This result is because of the trailing-edge flap discrepancy seen in the OBES comparisons. The AAW model does a very good job of predicting all the responses for this maneuver. 

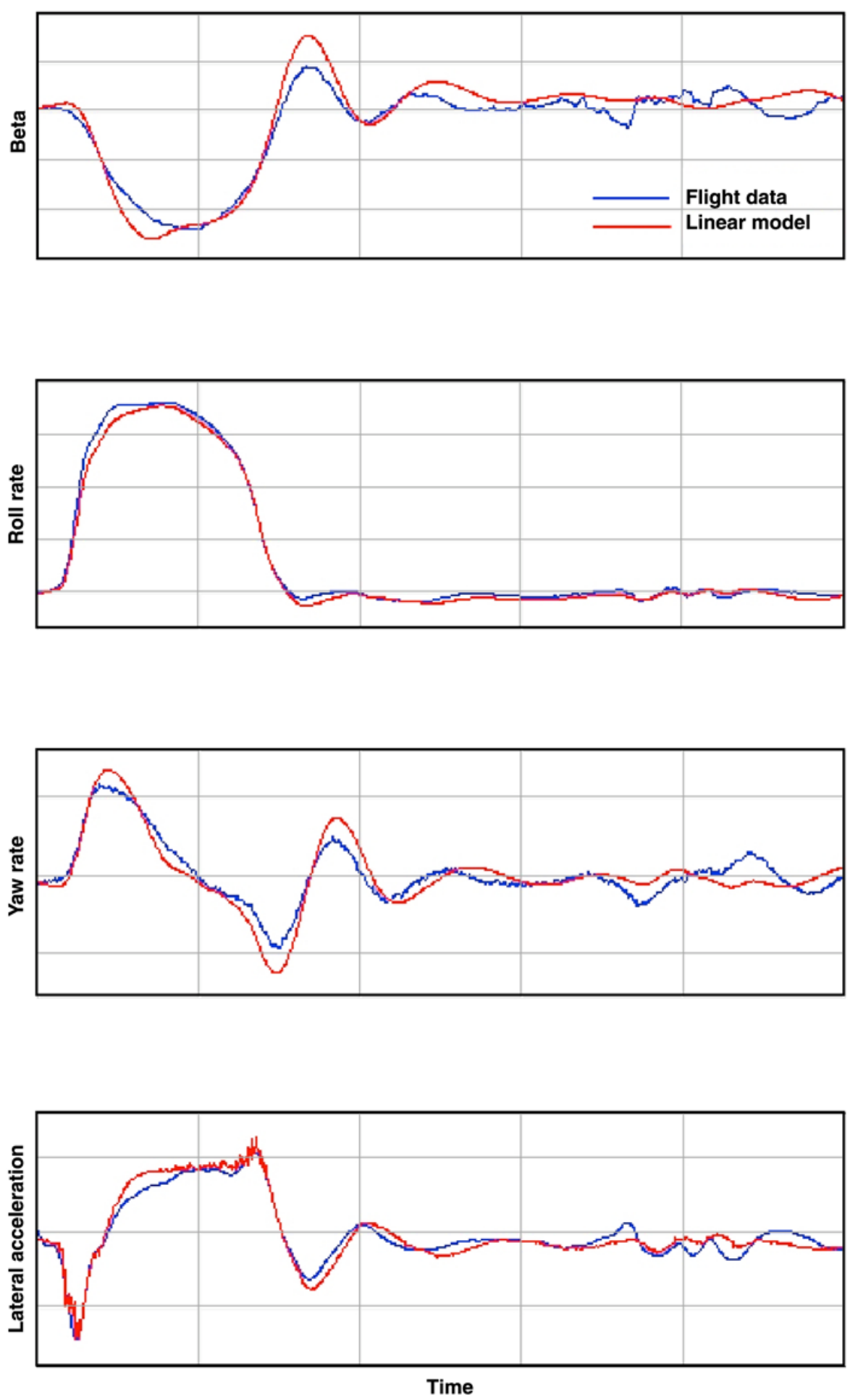

Figure 12. Linear aerodynamic model validation test. 

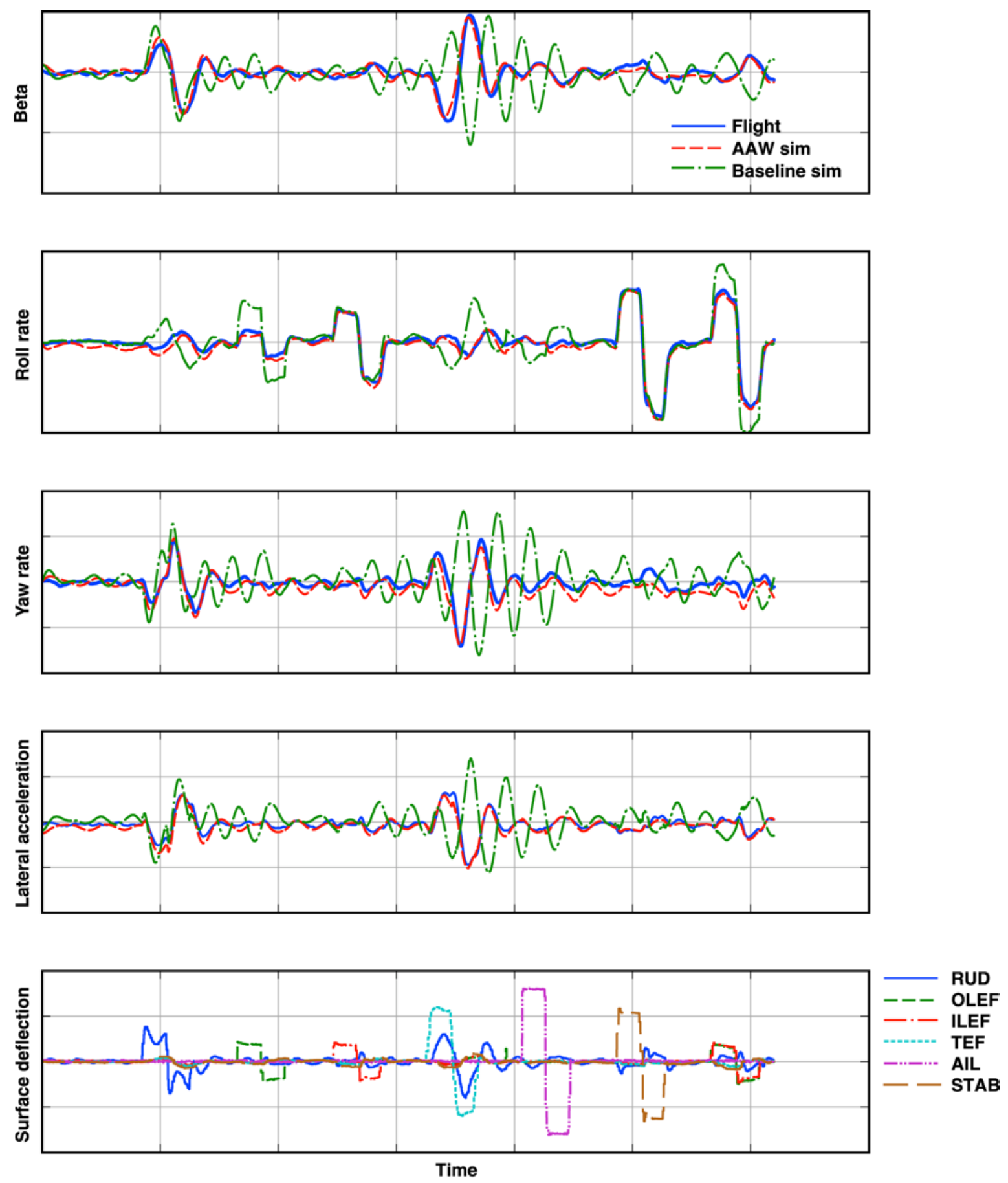

Figure 13. Open-loop lateral-directional Onboard Excitation System validation test at low-altitude, supersonic conditions. 

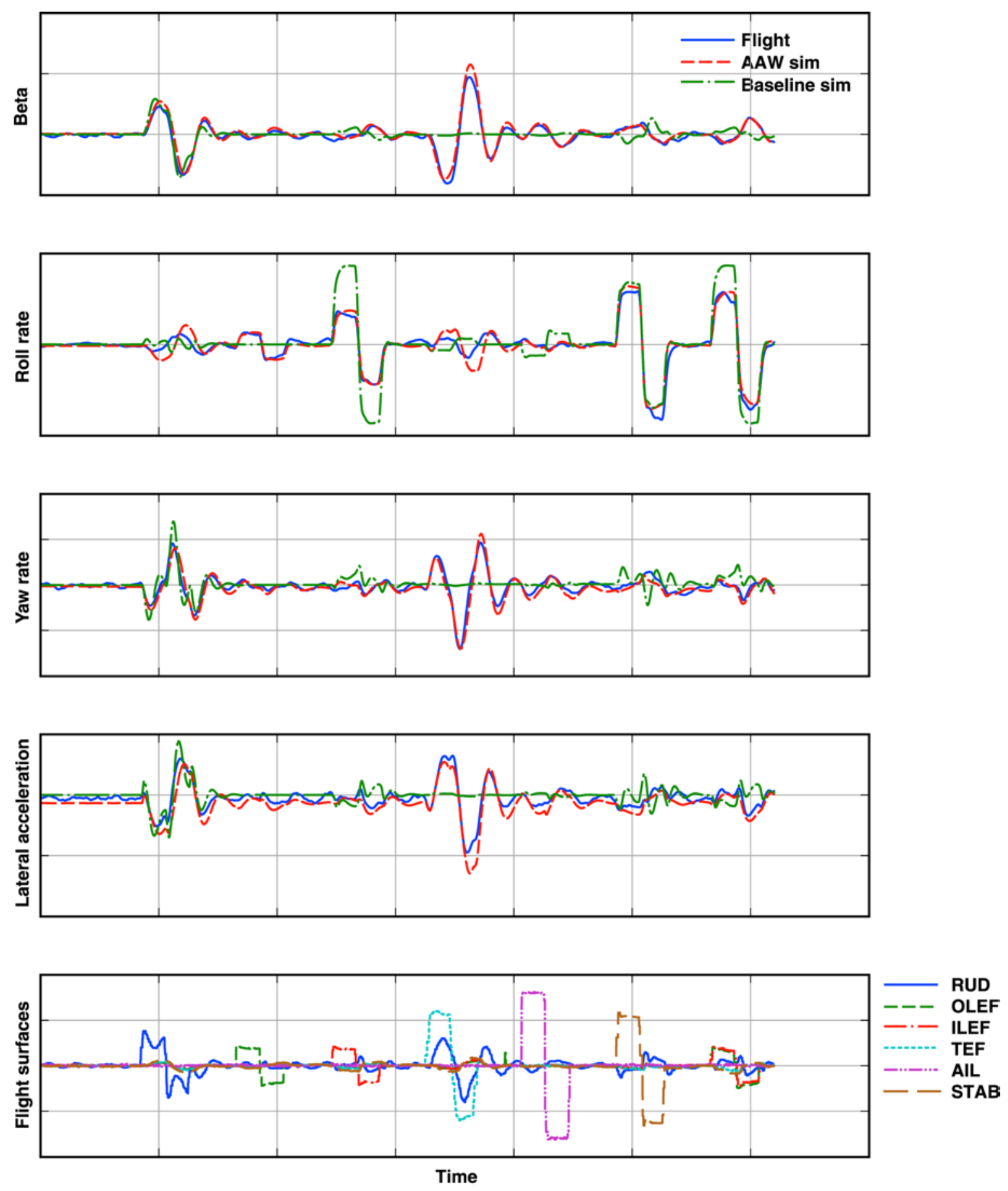

Figure 14. Closed-loop lateral-directional Onboard Excitation System validation test at low-altitude, supersonic conditions. 

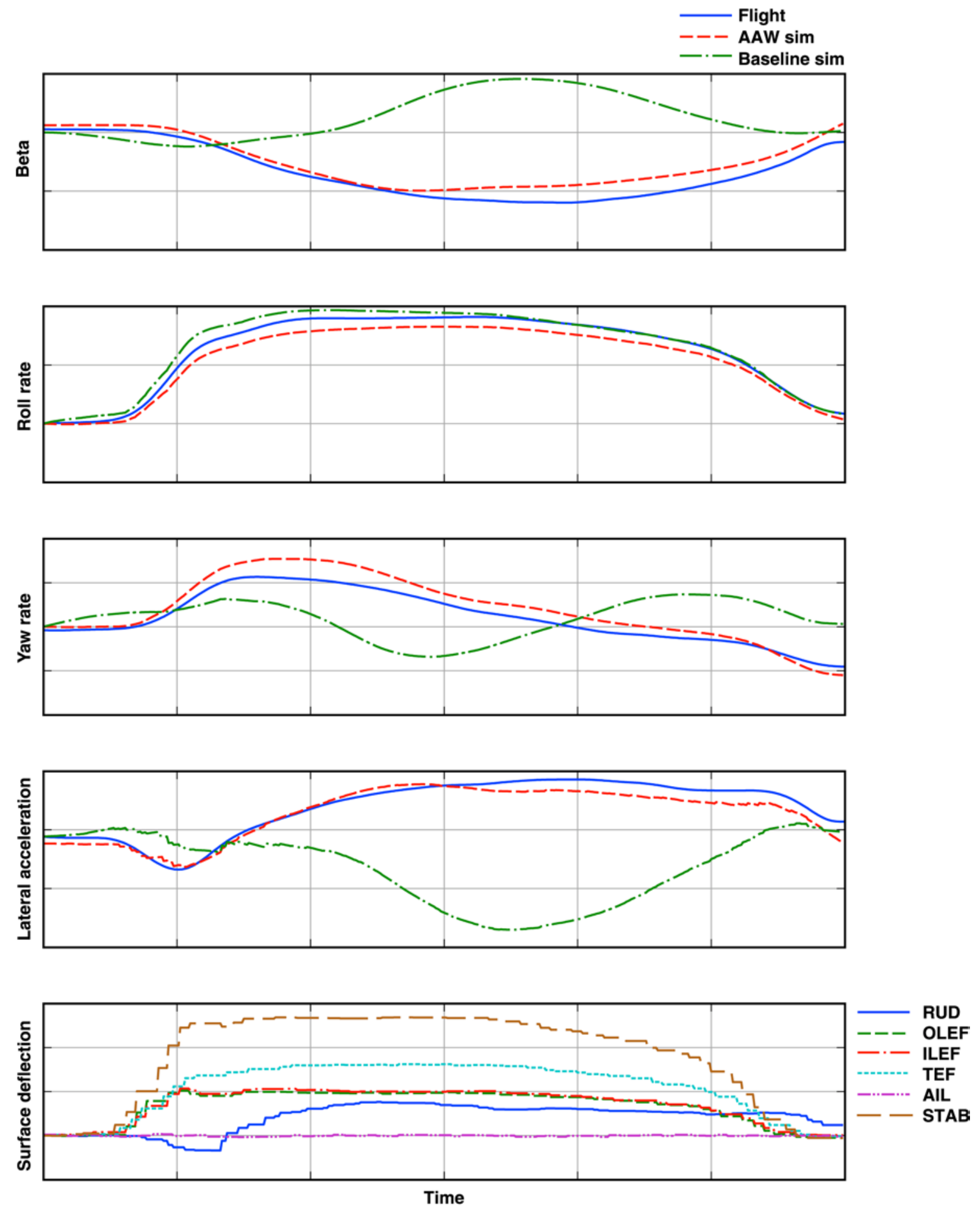

Figure 15. Open-loop integrated test block roll maneuver at low-altitude, supersonic conditions. 
Further aerodynamic model checkout was performed using the phase II flights.

\section{Phase II Flights}

Phase II consisted of 32 flights, flown with research control laws using RFCS. These control laws were flown at the 18 flight conditions previously discussed. The main research maneuvers performed during this phase were bank-to-banks, rolls, wind-up turns, and RPOs. These control laws were designed to take advantage of the additional control surface actuation available, creating wing twist to assist in rolling maneuvers. To produce rolling moments, deflections from all four of the wing surfaces could be used. These surfaces included ILEF, OLEF, TEF, and ailerons. Differential stabilators were not utilized. Standard F/A-18 control laws use differential stabilators in addition to the other surfaces to provide sufficient rolling moment. The AAW control laws were designed to create sufficient rolling moment without this surface deflection. Phase II also served as a means to verify the AAW model, as will be discussed.

Following the primary phase II flights, the research control laws for several flight conditions were replaced with new control laws. The flights following this control law change were referred to as phase IIa flights. These flights allowed researchers to examine different control laws at the same flight conditions.

\section{Aerodynamic Model Verification}

Informal verification of the AAW aerodynamic model was performed during phase II of the AAW project. This verification consisted of comparing flight data from various maneuvers, mainly rolling maneuvers, with the output from the simulation using the AAW model. Rolling maneuvers were the basis of the AAW project as well as the focus of the model verification. Results from bank-to-banks, 360-degree rolls, and RPOs were analyzed. Phase II verification was similar to the validation performed in phase I. In phase II, however, the control laws used for the maneuvers evaluated were designed to utilize the AAW concept. The phase II maneuvers, therefore, provided the opportunity for a comprehensive test of the aerodynamic model. Analysis of the results from these flight tests showed that the AAW model was an adequate aerodynamic model for use with these flight tests.

\section{A. Lateral-Directional Responses}

Roll rates predicted using the AAW aerodynamic model were very good overall when compared with flight data. The roll rates matched the flight trends throughout the maneuvers and often were nearly identical to the actual aircraft roll rate. Figure 16 shows an excellent roll rate match throughout the maneuver. Figure 17 shows a less accurate match. Here, the AAW model roll rate follows the same trend as the flight data, but with an under-prediction for the steady-state roll rate. For most flight conditions the steady-state roll rates for 360-degree rolls and bank-to-banks were under-predicted.

Yaw rates matched flight trends well overall, but not as well as roll rates. Figure 18 is an example of the AAW model matching the flight yaw rate throughout the course of the maneuver. Figure 19 shows a maneuver in which the AAW model simulation does not match the flight yaw rate closely. The matching is reasonable at the start of the maneuver, but as the maneuver progresses, the yaw magnitudes seen in flight are not well-predicted by the AAW model.

Lateral acceleration outputs from the AAW model were often offset from the lateral acceleration seen in flight. Though it matched closely for some maneuvers, such as in Fig. 18, often the lateral acceleration comparisons more closely resemble Figs. 16 and 20. Simulation trends match flight data very well, but the offset is persistent. At times the AAW simulation would not trim to the initial flight lateral acceleration. The resulting offset would continue, and in some cases, increase throughout the maneuver, as in Fig. 16. In other instances, the simulation would trim well at a steady-state condition, but would be offset from the flight data throughout the actual maneuver, as in Fig. 20. This offset, while often present, is generally of a magnitude small enough to be insignificant.

Beta was a particularly important parameter in control law design for AAW as well as during the flights. Excessive sideslip during rolls could result in a departure from controlled flight. Beta prediction by the AAW model was generally adequate. Beta did not match trends as well as some of the other lateral-directional responses, but still matched general trends reasonably well. Figure 17 is a good example of a reasonable match without near-perfect trend matching. Figure 21 is a good example of beta from the AAW simulation matching the flight trends well throughout the maneuver. 

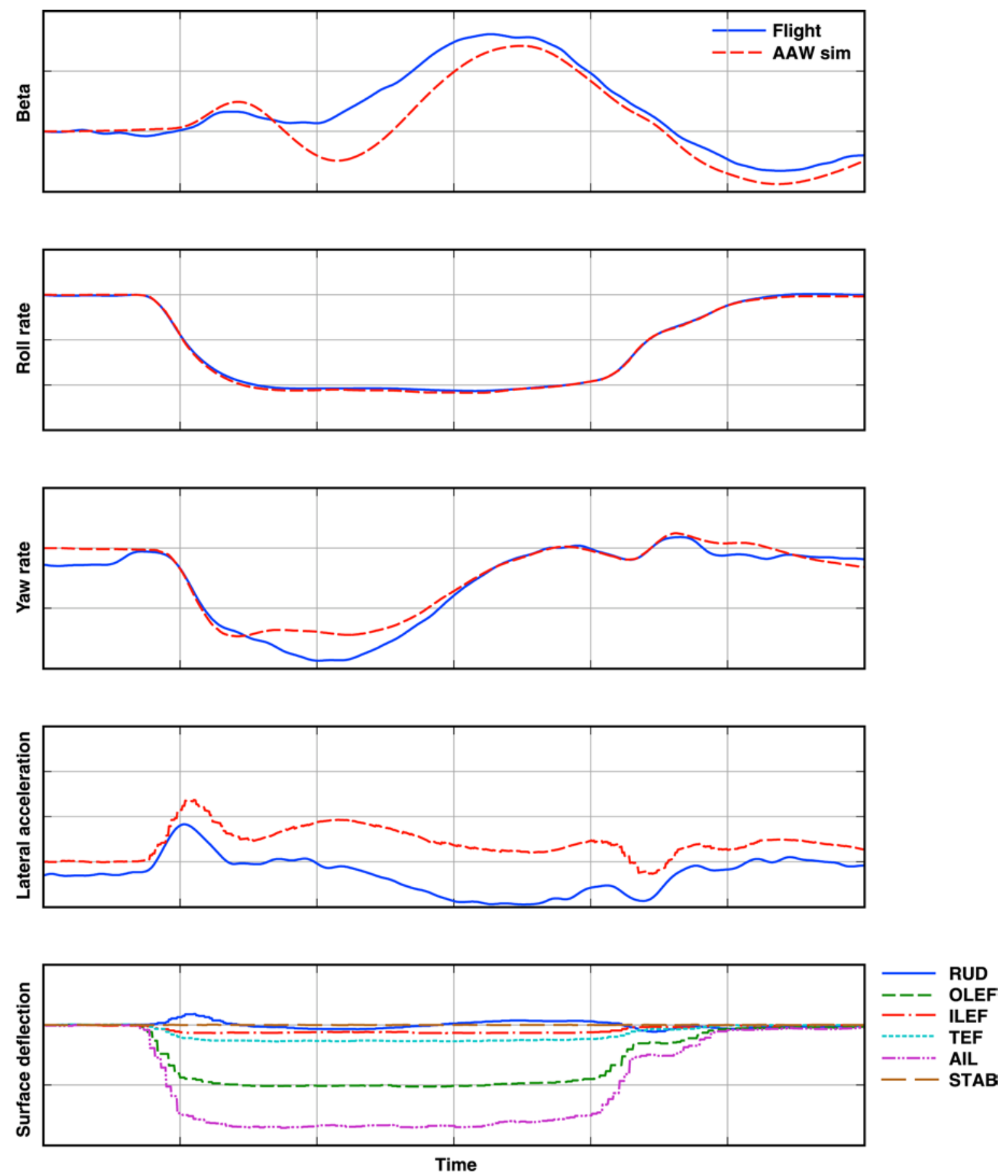

Figure 16. Lateral-directional responses from a 75-percent stick left roll at low-speed subsonic conditions. 

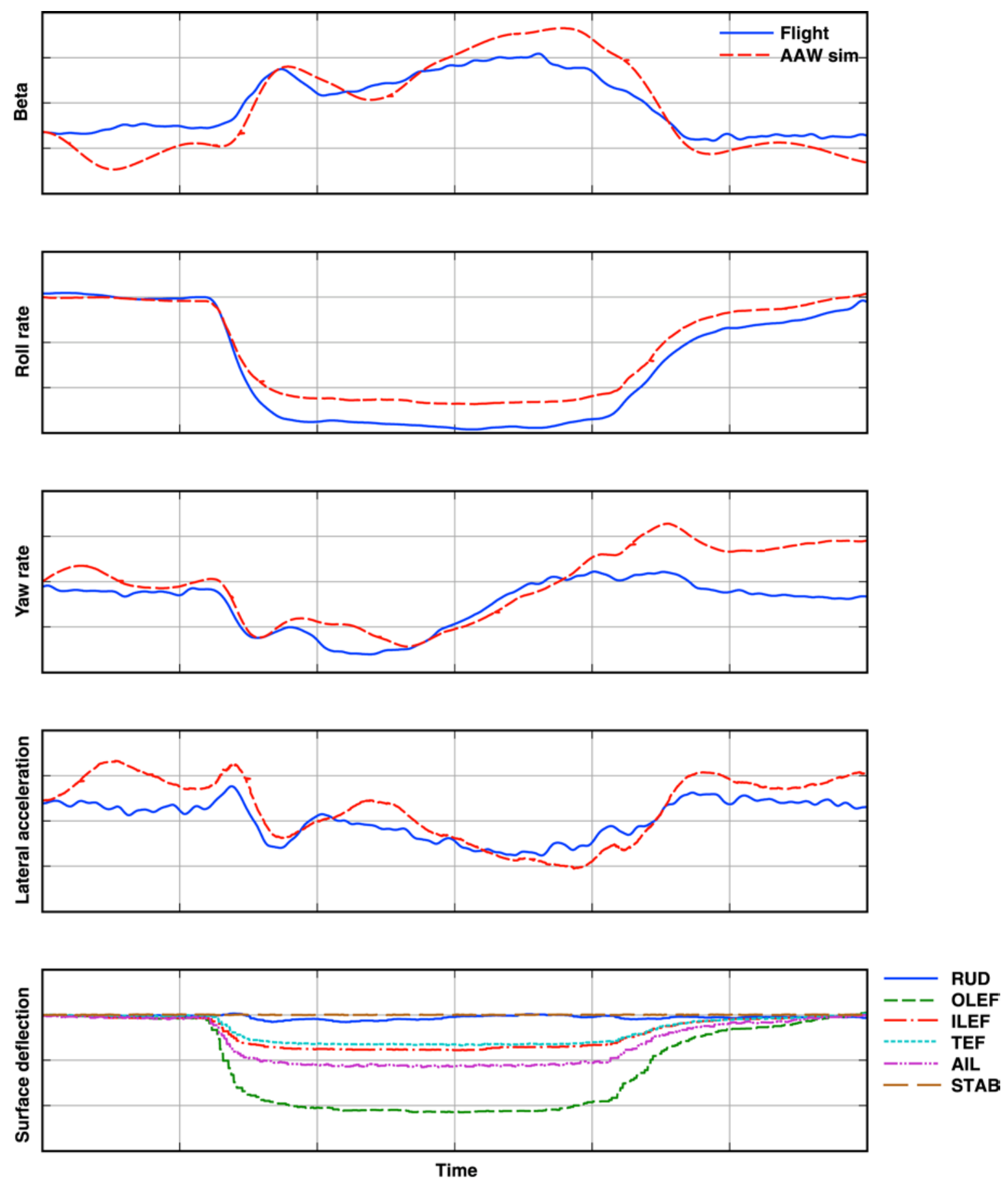

Figure 17. Lateral-directional responses from a 75-percent stick left roll at high-speed subsonic conditions. 

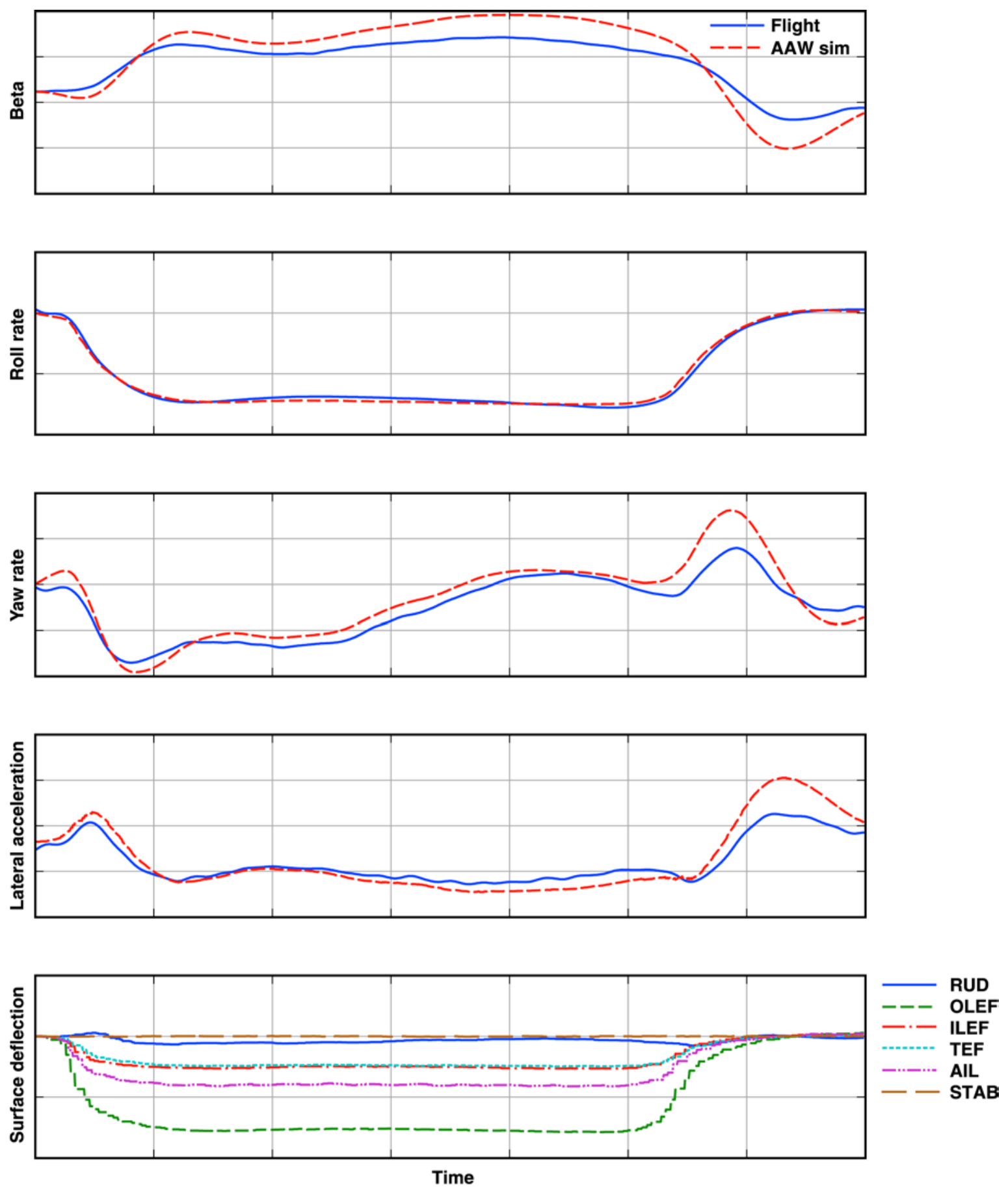

Figure 18. Lateral-directional responses from a full-stick left roll at high-speed subsonic conditions. 

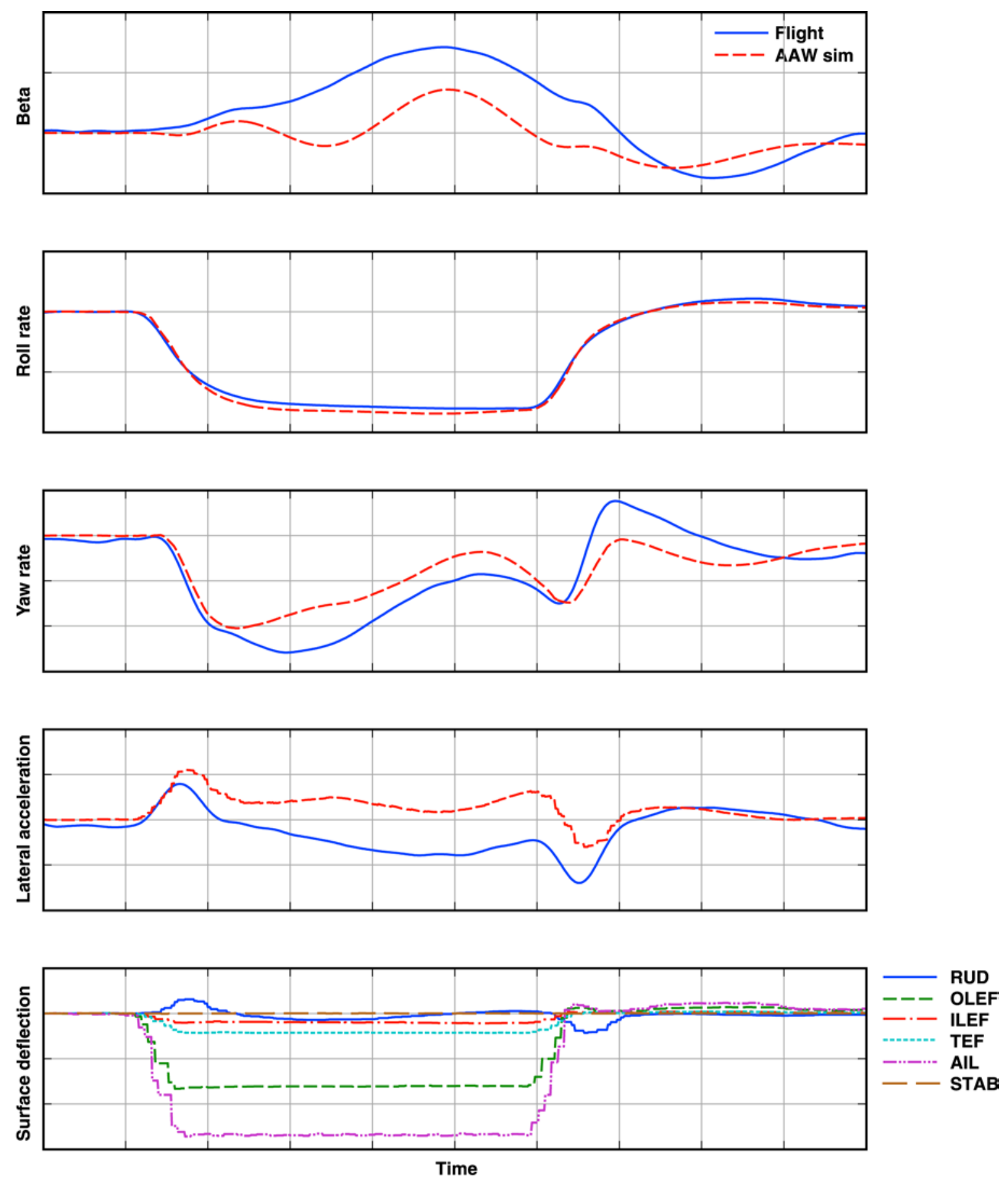

Figure 19. Lateral-directional responses from a full-stick left roll at low-speed subsonic conditions. 

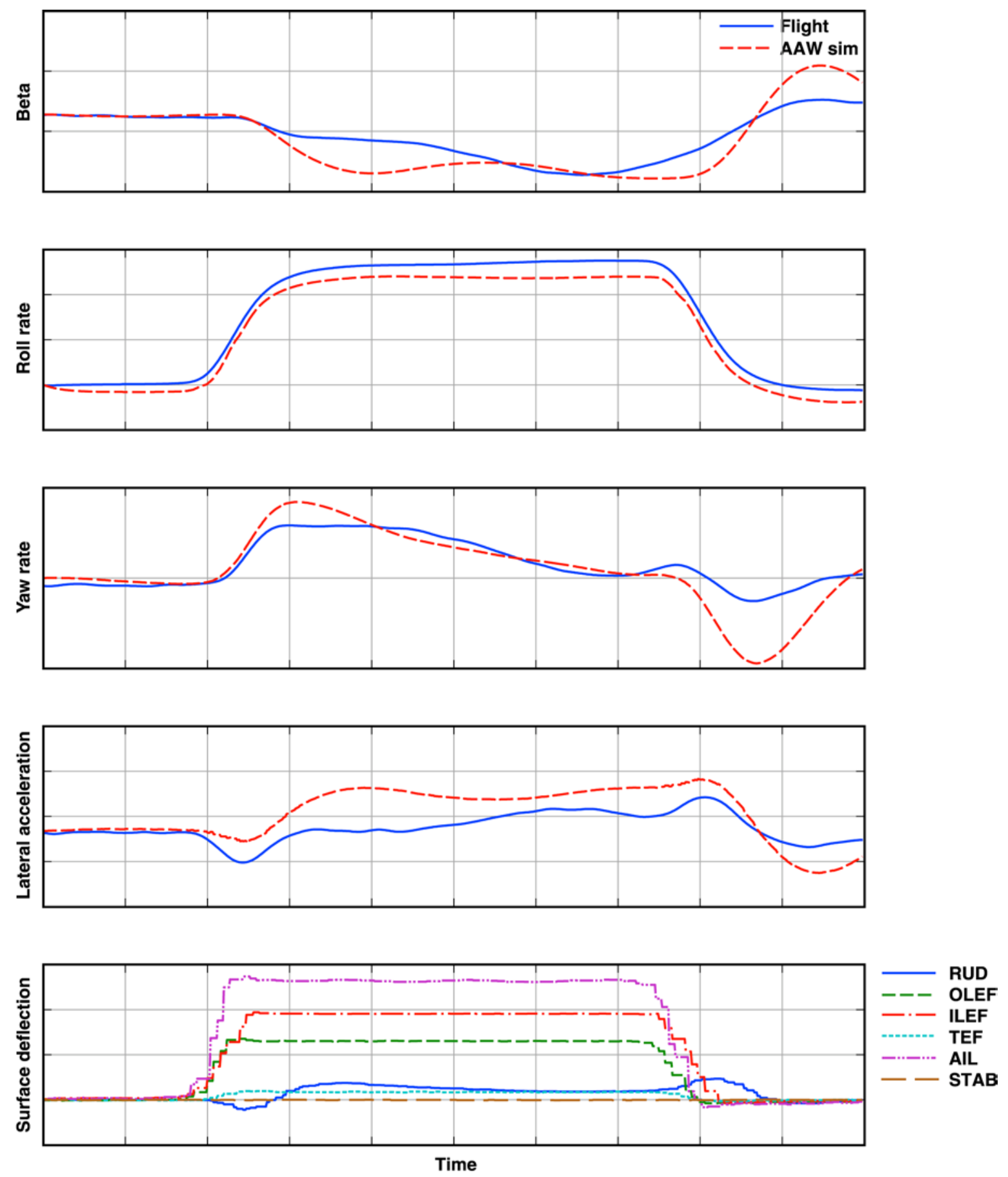

Figure 20. Lateral-directional responses from a full-stick right roll at low-altitude, supersonic conditions. 

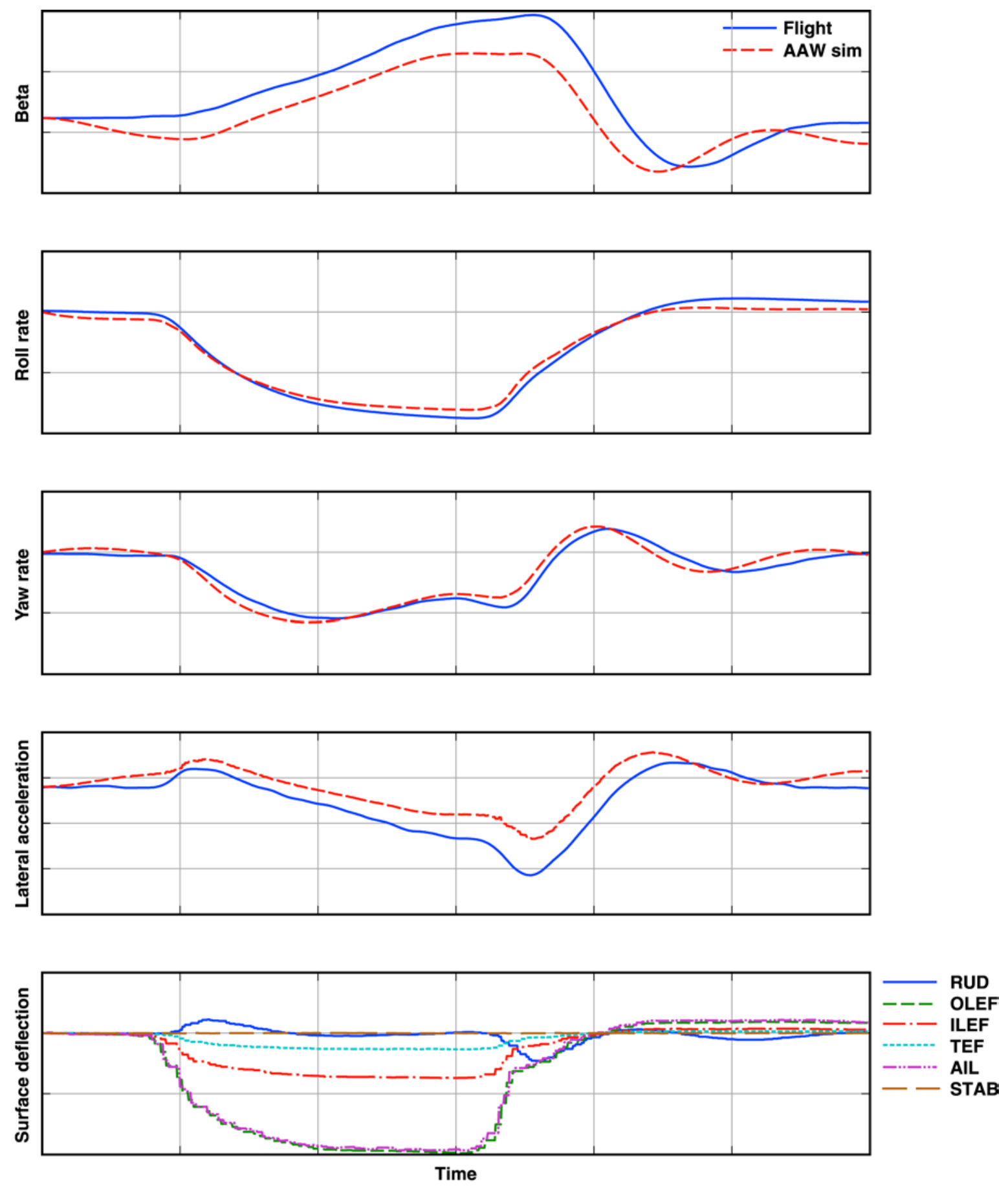

Figure 21. Lateral-directional responses from a 75-percent stick left roll at high-altitude, supersonic conditions. 


\section{B. Longitudinal Responses}

The longitudinal responses from the AAW aerodynamic model matched the flight data reasonably well for rolling maneuvers. While the simulation did not always trim to the flight values, the outputs generally followed the flight data trends. Figure 22 shows flight data and AAW model simulation data for the longitudinal responses from a subsonic roll maneuver. As can be seen in the figure, the longitudinal time history from the simulation matches the trends of the flight data. Figure 23 shows a supersonic roll maneuver. Again, the AAW simulation follows the flight data trends reasonably well considering many of the responses result from cross-coupling. Angle of attack is under-predicted for a portion of the maneuver because of the slight under-prediction in the negative pitch rate spike that occurs during the roll. Considering that cross-coupling factors were not included in the aerodynamic model, the longitudinal responses for lateral-directional maneuvers matched flight trends reasonably well.
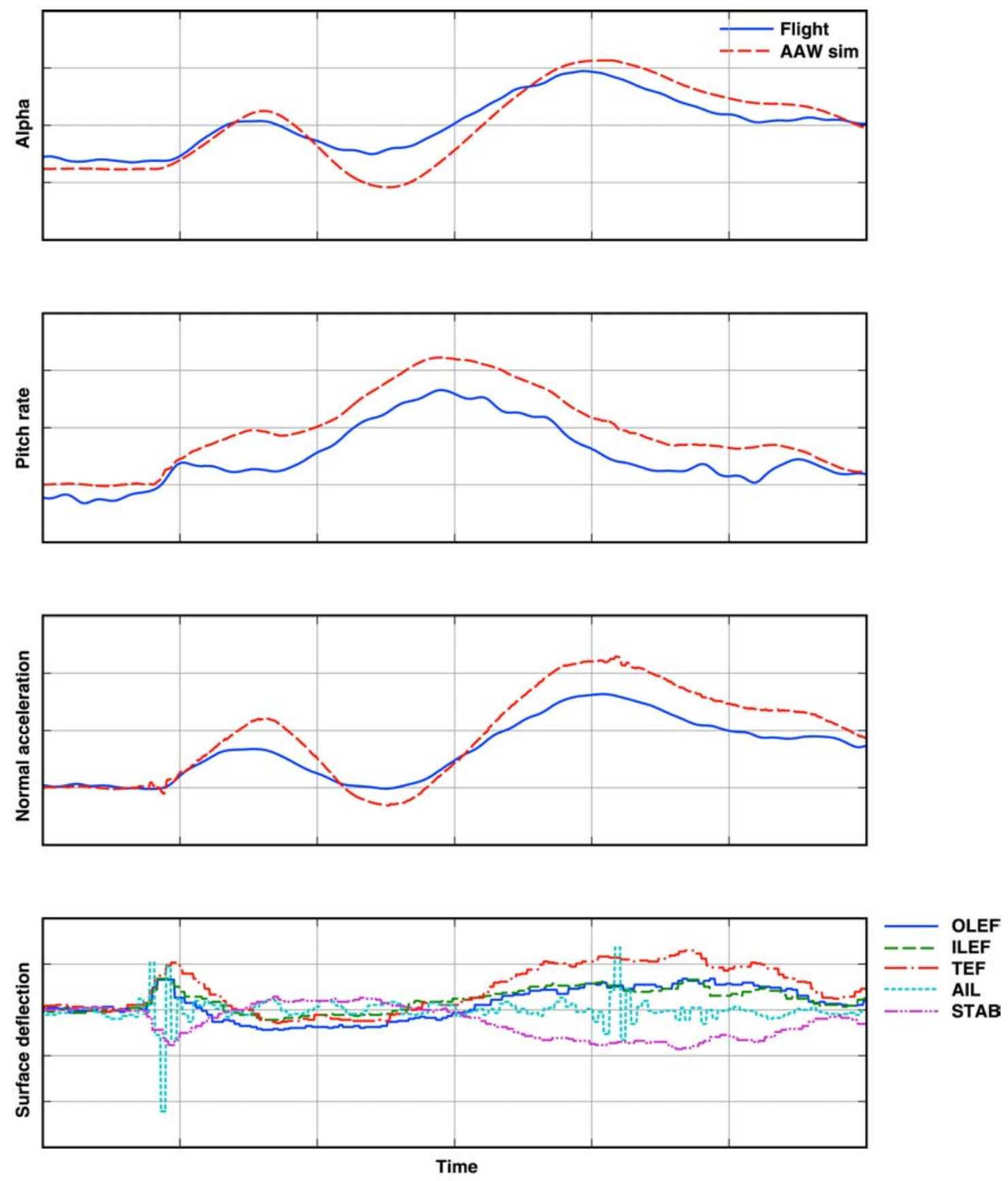

Figure 22. Longitudinal responses from a 75-percent stick left roll at low-speed subsonic conditions. 

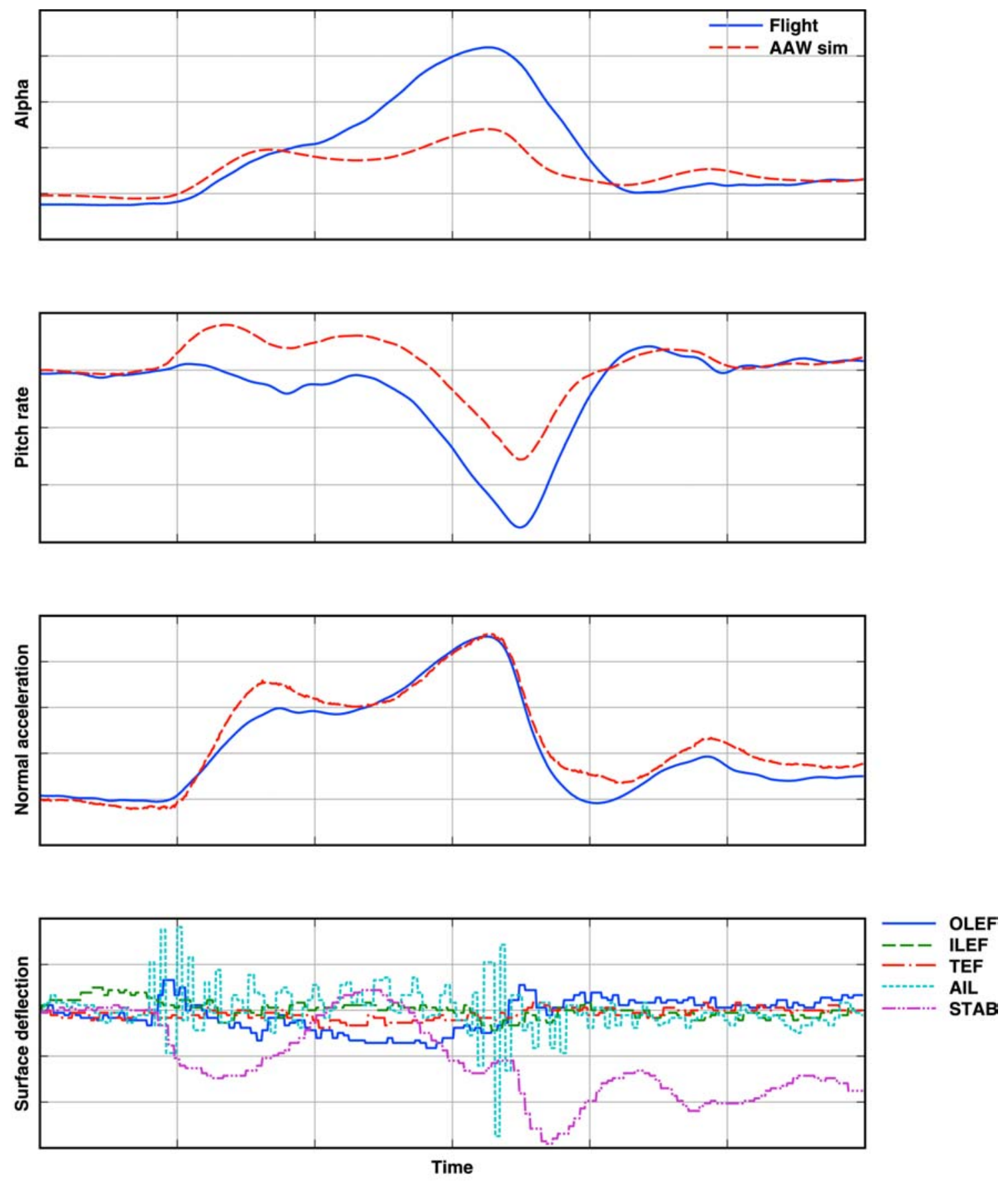

Figure 23. Longitudinal responses from a 75-percent stick left roll at high-altitude, supersonic conditions. 


\section{Effects of Maneuver Variations}

The agreement between the flight data and the simulation results using the AAW model remained reasonable regardless of the magnitude or direction of stick input. Trends were followed reasonably for the various stick inputs, including rolls initiated by various magnitude lateral stick deflections. Figures 24, 17, and 18 show half-stick, 75-percent stick, and full-stick left rolls. While the AAW simulation results in all three figures do not match flight data identically, the predictions do match flight data trends reasonably. For this specific flight condition, the roll rate matching appears best for the full-stick maneuver. While this result is found in some flight conditions, it is not a consistent trend. Figure 25 shows a full-stick right roll at the same test conditions. The AAW simulation does not match the flight data identically for the two maneuvers, but trends are followed reasonably for both. While rolls in different directions did not result in identical matching, the AAW aerodynamic model proved to be adequate regardless of roll direction.

Rolling pull-outs were performed to examine the effects of loading on roll performance. The AAW model did a reasonable job predicting performance for these maneuvers at supersonic flight conditions. Subsonic RPOs were not analyzed for this report. Figure 26 shows a supersonic RPO with the same stick deflection as Fig. 20 (75-percent stick right). The matching is similar in this maneuver as it was for the 360-degree roll. The RPOs more consistently had simulation results offset from the flight data for steady-state roll rate than did unloaded rolls. While the consistency of this offset is notable, the magnitude is not significantly larger than what was seen for 360-degree rolls. Figure 27 shows another example of a supersonic RPO. Again, the matching is similar to that found in Fig. 21, a roll at the same flight conditions and stick magnitude, but in the opposite direction (left). Longitudinal responses from the simulation for the RPOs generally matched flight data well. Figure 28 shows the longitudinal responses for an RPO. The simulation outputs match flight data reasonably well. 

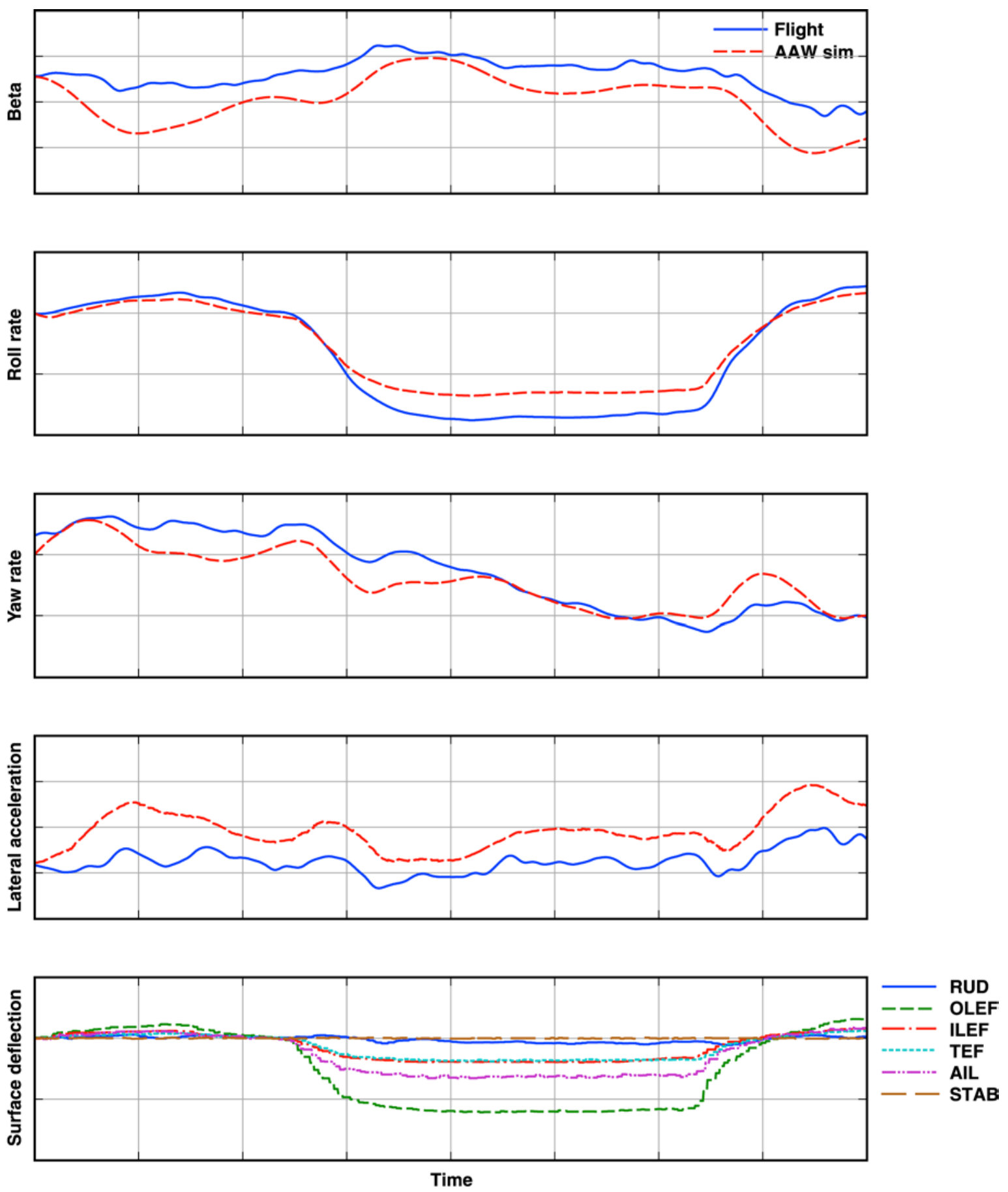

Figure 24. Lateral-directional responses from a half-stick left roll at high-speed subsonic conditions. 

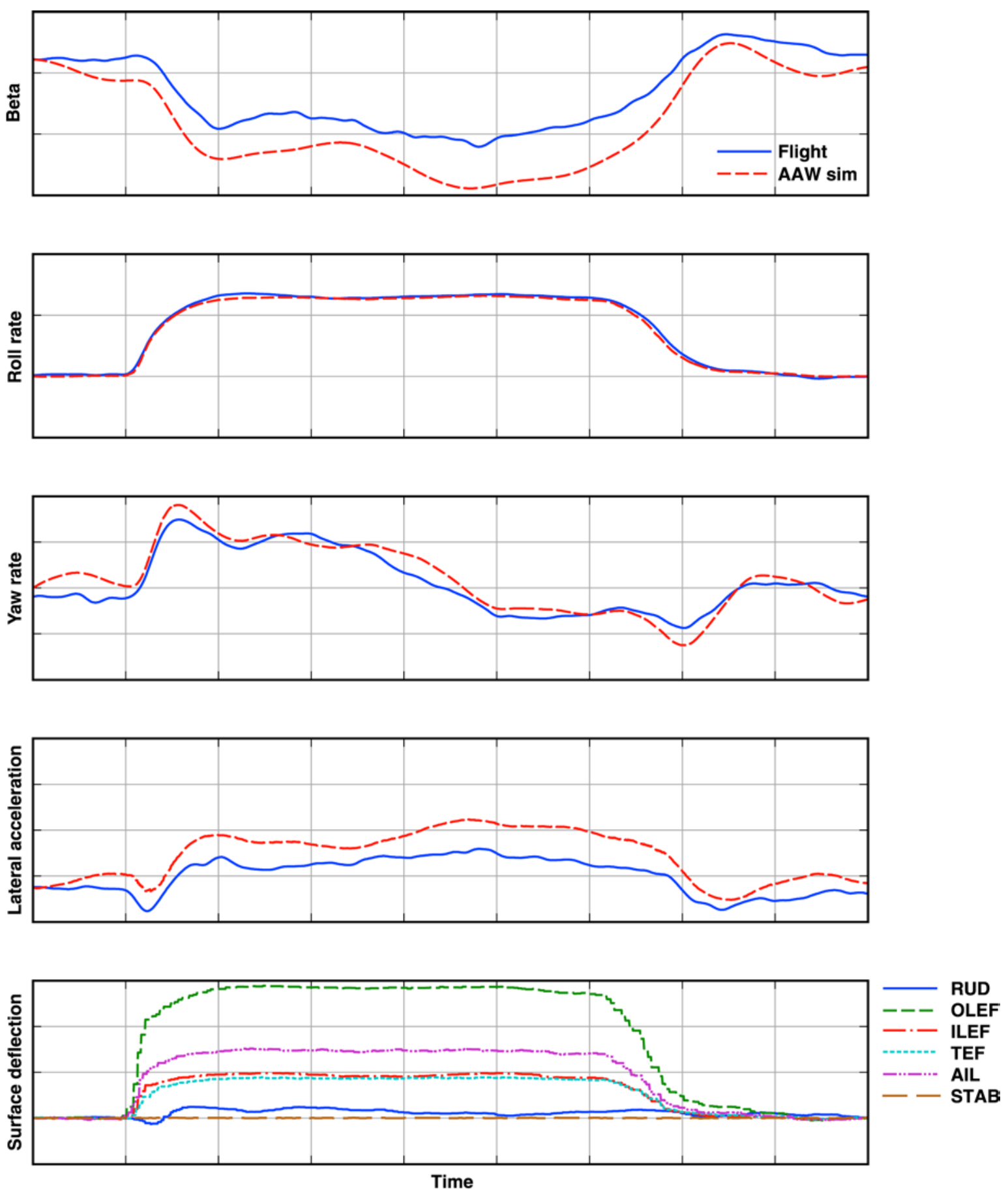

Figure 25. Lateral-directional responses from a full-stick right roll at high-speed subsonic conditions. 

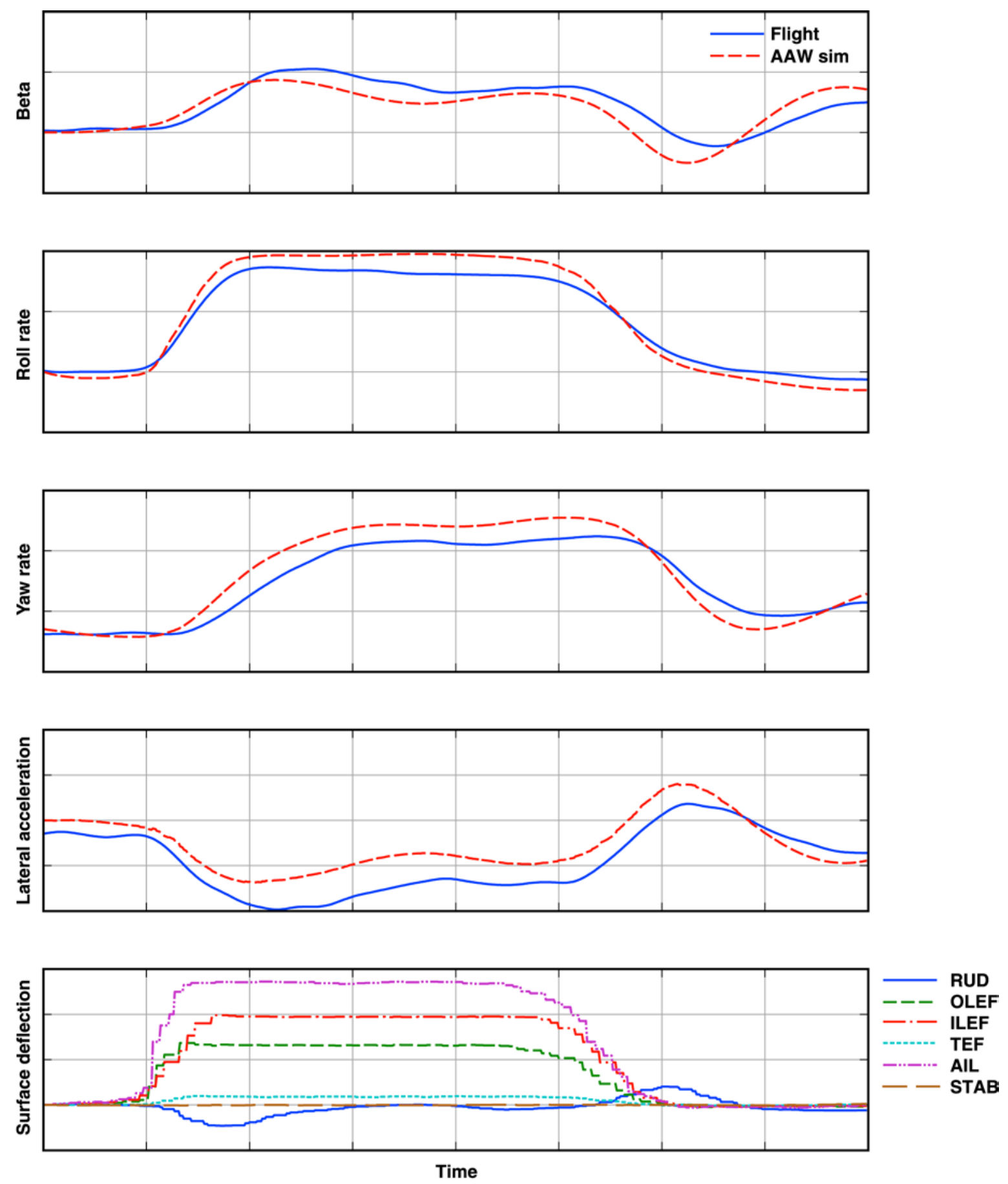

Figure 26. Lateral-directional responses from a full-stick right rolling pull-out at low-altitude, supersonic conditions. 

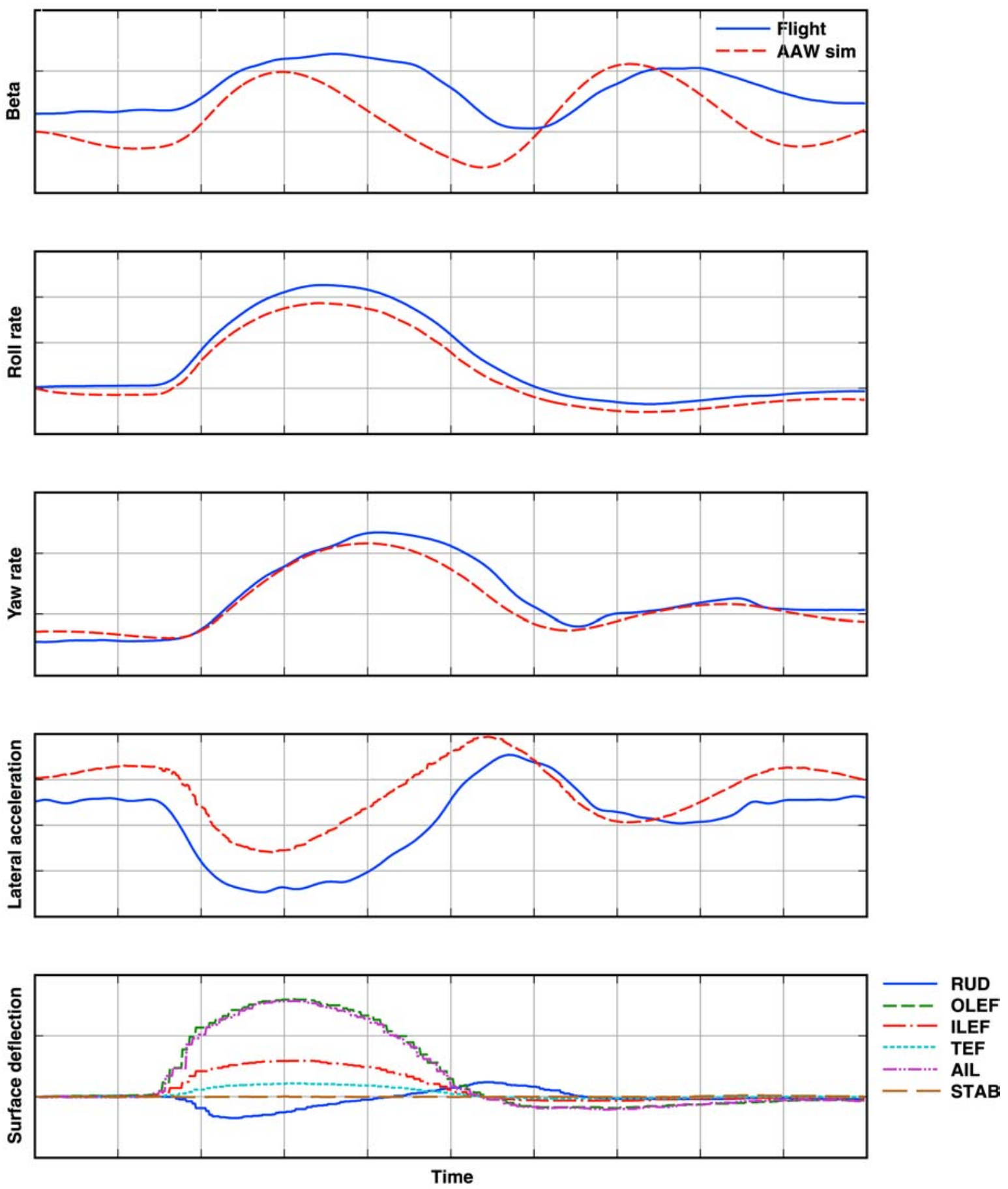

Figure 27. Lateral-directional responses from a 75-percent stick right rolling pull-out at high-altitude, supersonic conditions. 

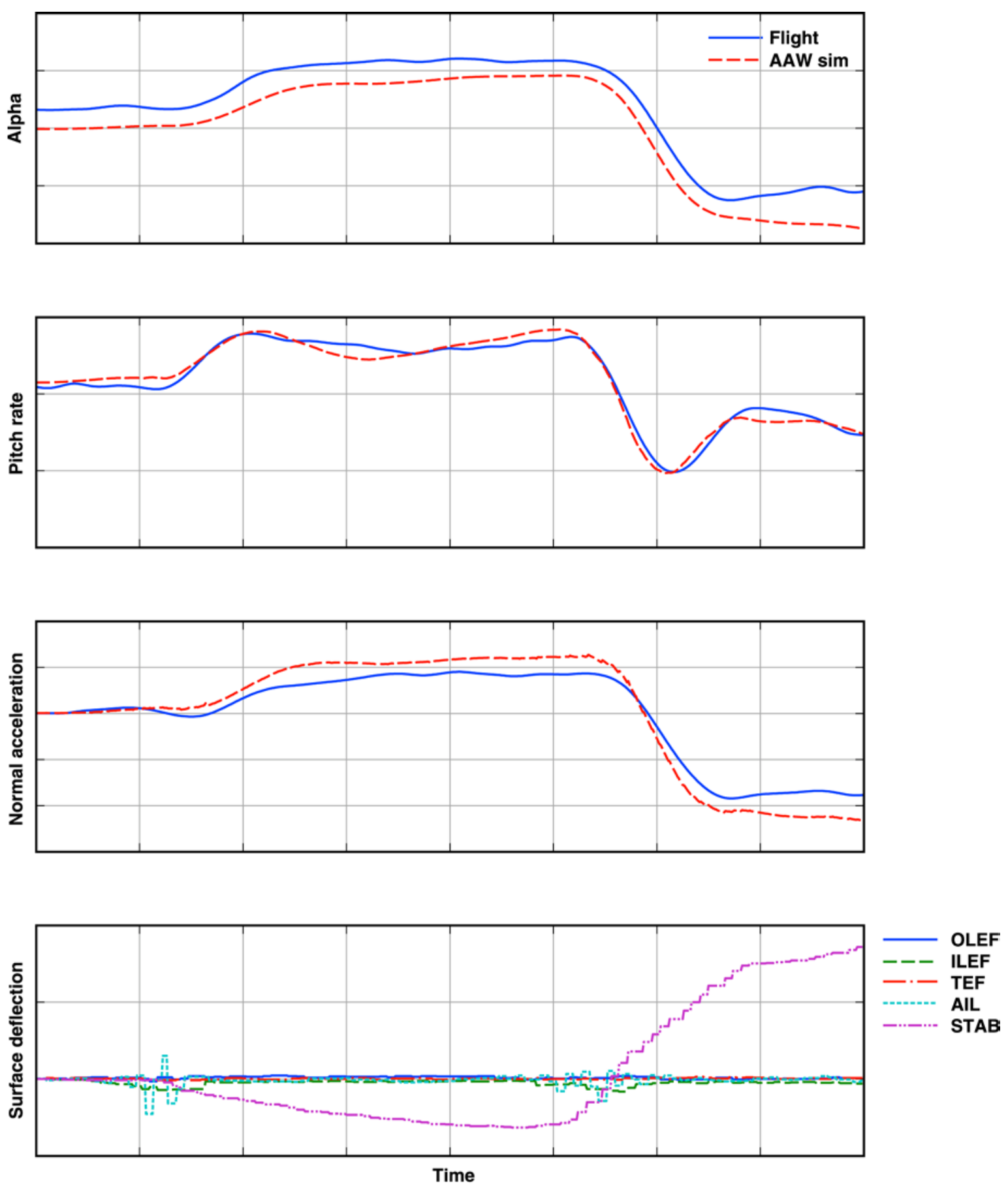

Figure 28. Longitudinal responses from a full-stick right rolling pull-out at low-altitude, supersonic conditions. 


\section{Phase IIa}

During the portion of the project informally known as phase IIa, new control laws were implemented for several of the flight conditions, and maneuvers were performed utilizing these control laws. These control laws used the available surfaces differently than did the phase II control laws. Examples of maneuvers using the phase IIa control laws can be seen in Figs. 29 and 30. Figures 17 and 21, respectively, are roll maneuvers flown with the same stick deflection using the phase II control laws. A comparison of the differential surfaces between the two figures from the same flight conditions illustrates the differences in control surface allocation. The degree to which the simulation matches flight data does not vary significantly for these maneuvers. This result demonstrates that the accuracy of the aerodynamic model is not dependent upon the control laws used or surfaces actuated.
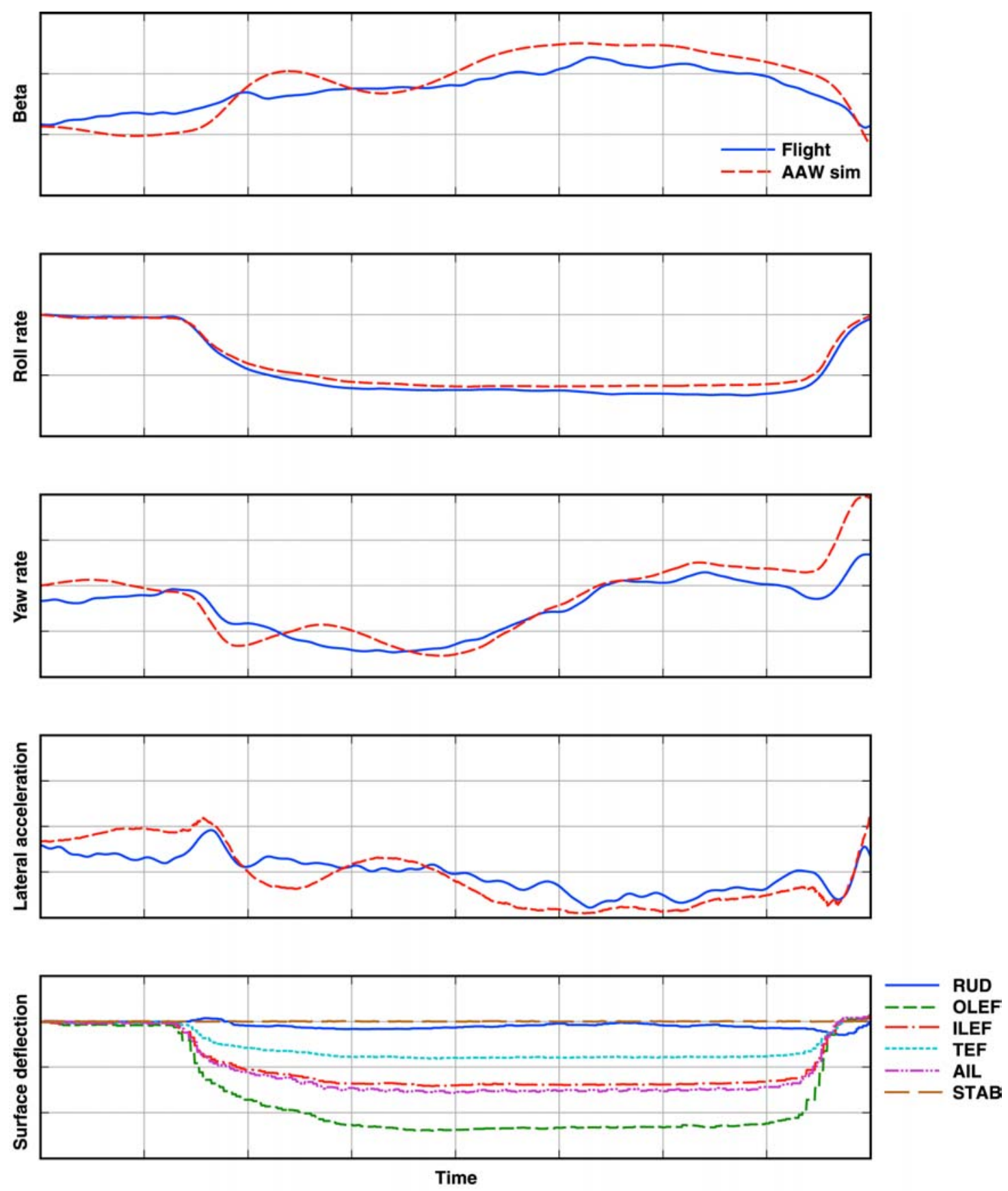

Figure 29. Lateral-directional responses from a 75-percent stick left roll at high-speed subsonic conditions (phase IIa control laws). 

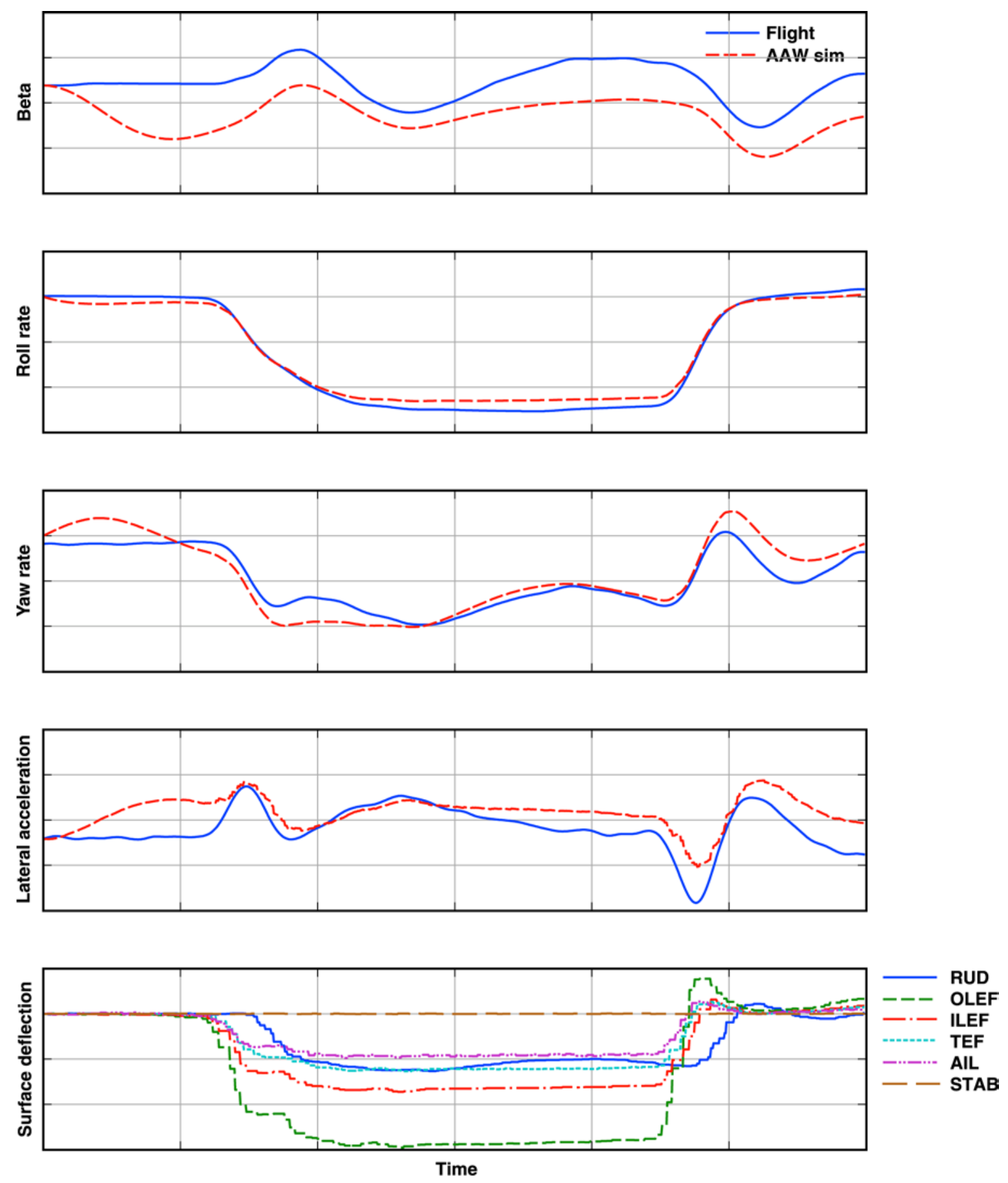

Figure 30. Lateral-directional responses from a 75-percent stick left roll at high-altitude, supersonic conditions (phase IIa control laws). 


\section{E. Comparison with Baseline}

A comparison of the AAW aerodynamic model with the baseline F-18 model clearly shows that the AAW model is superior for the AAW aircraft flying at applicable flight conditions. While the baseline model did a reasonable job for some flight conditions, it rarely outperformed the AAW model. Maneuvers previously discussed solely in the context of the AAW model will now be shown with both the AAW and the baseline model.

Figures 31 through 34 show comparisons between flight data, the AAW model and the baseline model. Figure 31 is an example of the baseline model matching flight beta reasonably, but poorly matching the roll rate from flight. Yaw rate and lateral acceleration from the baseline model do not match flight data particularly well. Figure 32 shows another subsonic roll maneuver. In this instance, beta and lateral acceleration outputs from the baseline simulation trend the opposite direction from flight and the AAW simulation outputs. The yaw rate from the baseline model is reasonable and the roll rate is nearly as accurate as the AAW model. Figure 33 shows a supersonic maneuver. Similarly to the subsonic maneuver, beta and lateral acceleration trend in opposite directions from the flight data and AAW model. Again, roll rate and yaw rate from the baseline model match flight data reasonably, but do not match as closely as those from the AAW model. Figure 34 shows a supersonic RPO. Here, the baseline simulation trends similarly to the AAW simulation, but is farther away from the flight data for every lateral-directional response. Figure 35 illustrates a typical set of longitudinal responses from a roll for the baseline model. The baseline model does a reasonable job, but does not match flight data as well as the AAW model. From these figures, it is clear that the AAW aerodynamic model is an improvement over the baseline aerodynamic model. 

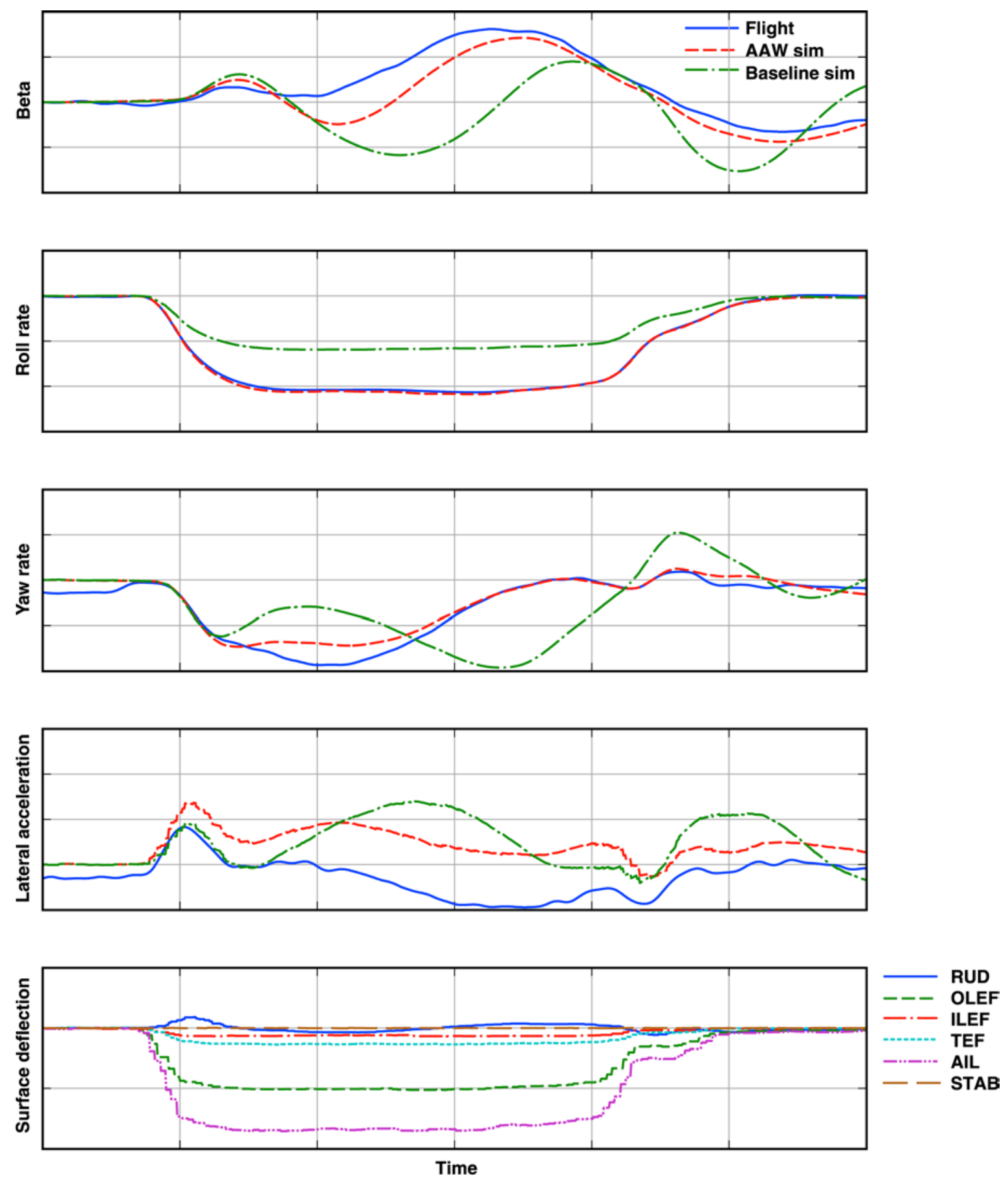

Figure 31. Lateral-directional responses from a 75-percent stick left roll at low-speed subsonic conditions. 

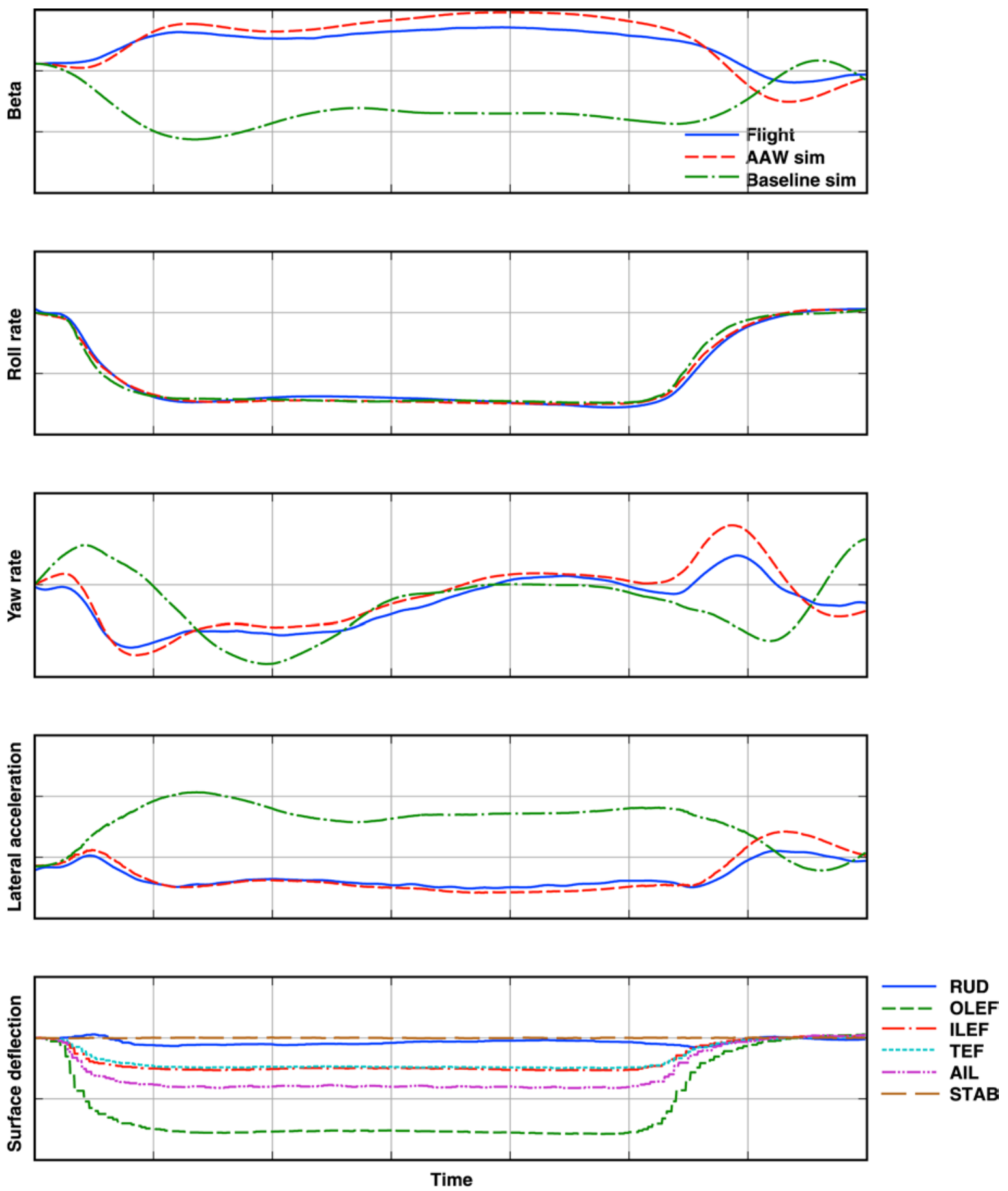

Figure 32. Lateral-directional responses from a full-stick left roll at high-speed subsonic conditions. 

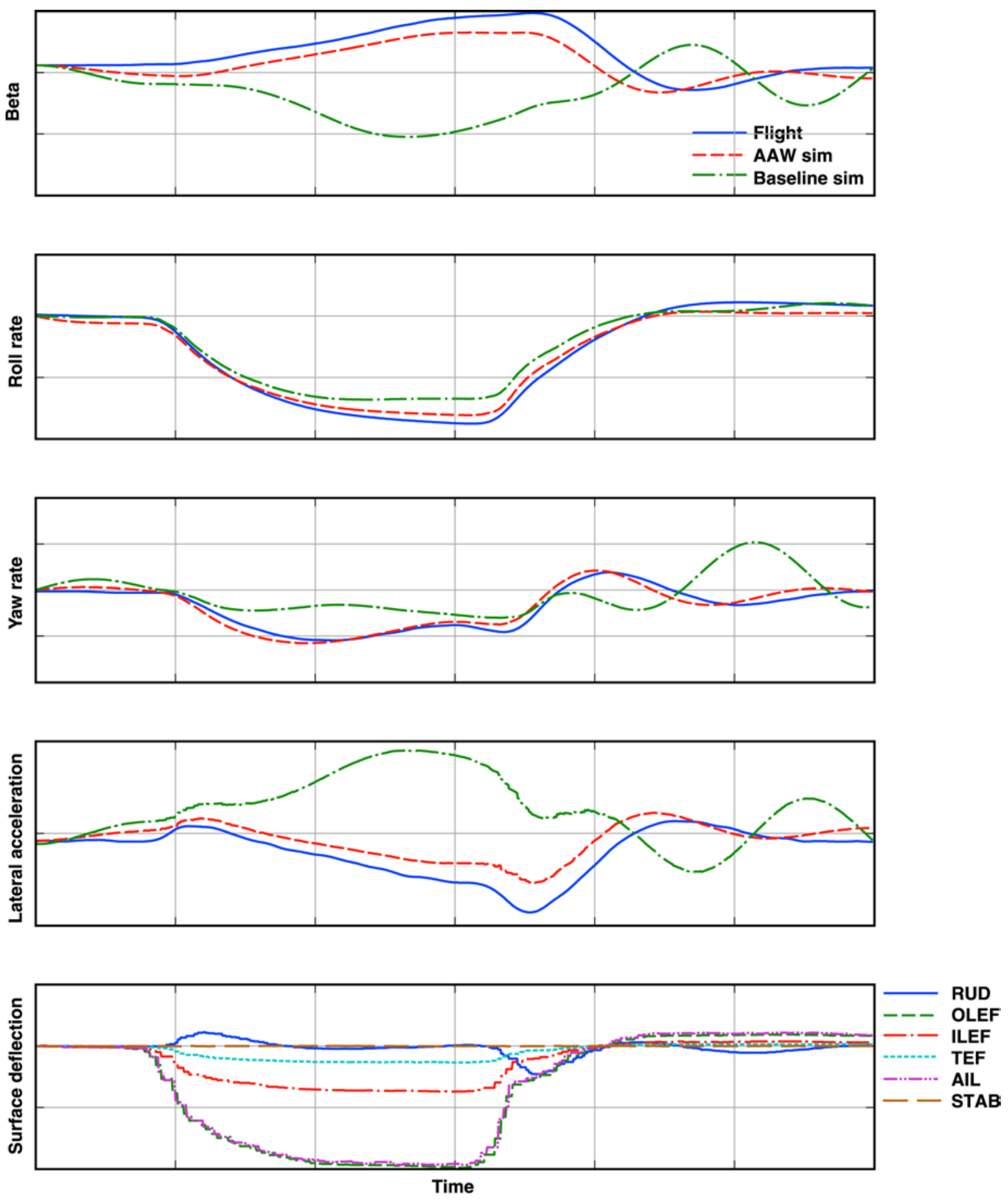

Figure 33. Lateral-directional responses from a 75-percent stick left roll at high-altitude, supersonic conditions. 

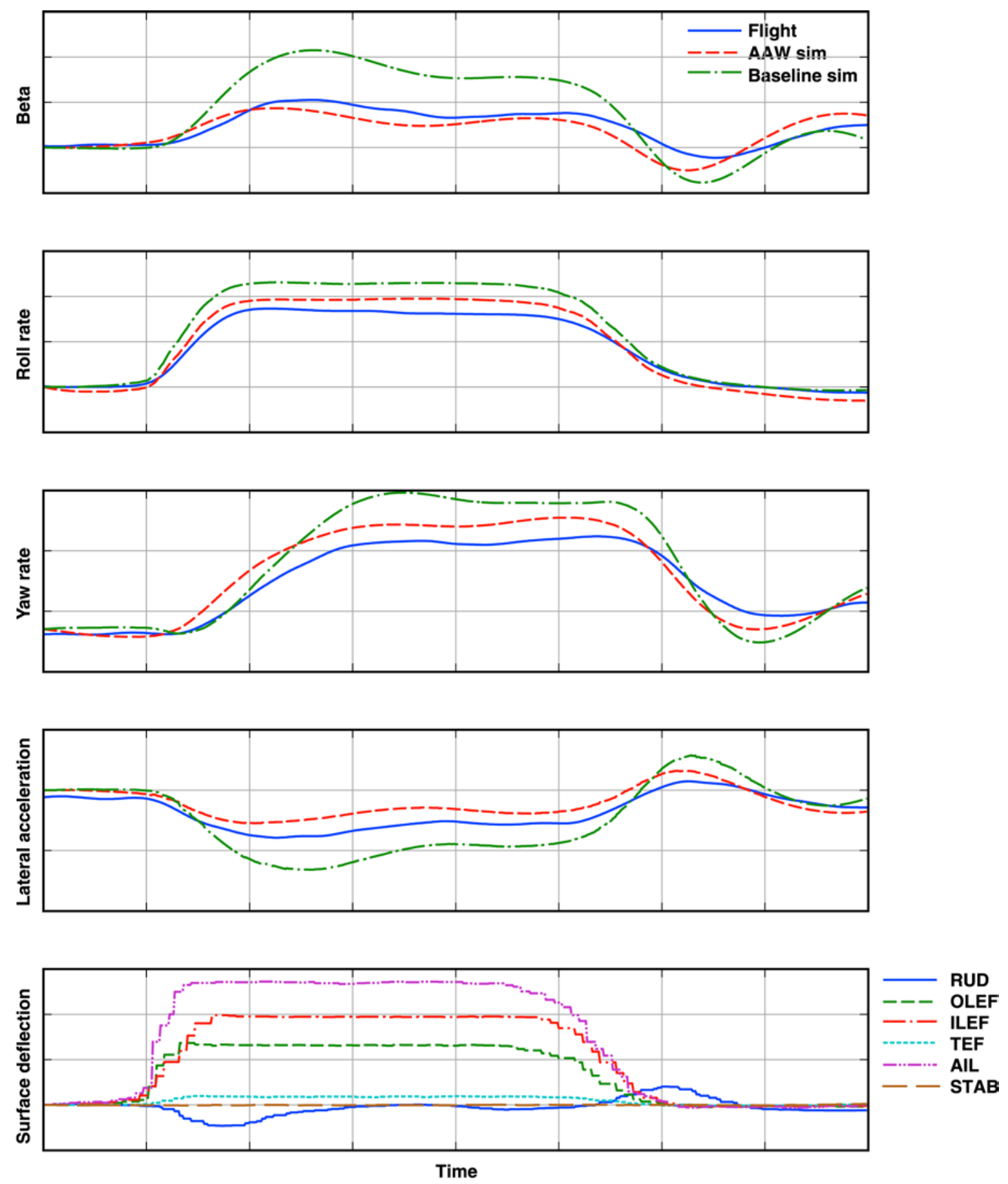

Figure 34. Lateral-directional responses from a full-stick right rolling pull-out at low-altitude, supersonic conditions. 

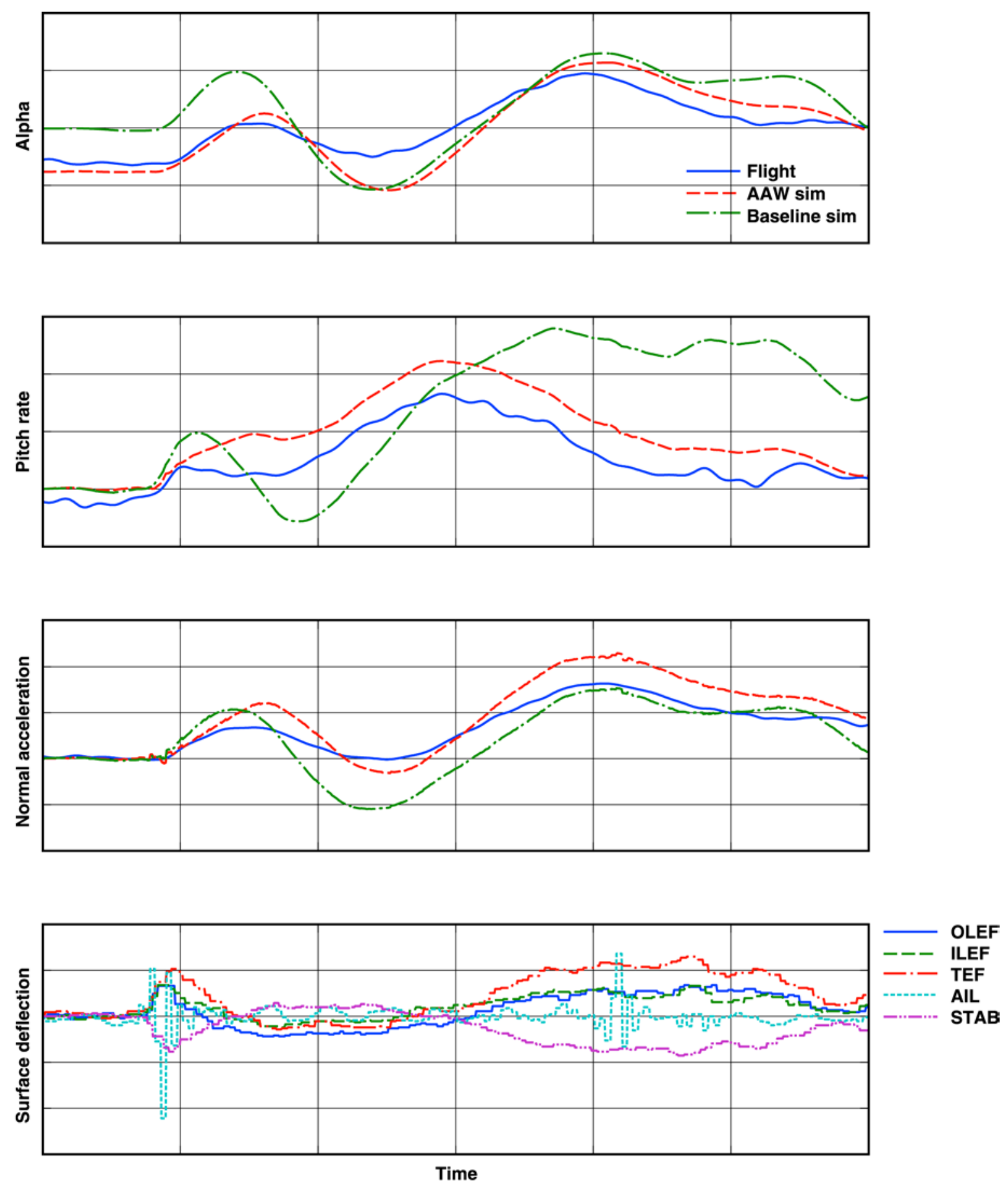

Figure 35. Longitudinal responses from a 75-percent stick left roll at low-speed subsonic conditions. 


\section{Conclusion}

A new aerodynamic model has been developed and validated for a modified F/A-18A used for the Active Aeroelastic Wing (AAW) research program. Results from the parameter identification analysis conducted for the AAW program have shown that existing F/A-18 aerodynamic models have several flaws and could be substantially improved through extensive parameter identification testing. The output-error technique discussed in this report has proven to be effective in providing linear stability and control estimates from flight data. It is recommended, however, that large coordinated maneuvers be used in conjunction with individual control surface inputs to ensure that estimates are applicable to a wide range of maneuvers. It is also necessary to thoroughly validate the model to identify any limitations.

The AAW aerodynamic model produced simulation data that compared well with flight data. Significant improvement over the baseline model was shown. The accuracy of the model did not vary significantly with control surfaces used. The model also performed well for loaded maneuvers, such as rolling pull-outs. Sideslip excursions not predicted by the baseline model were evident in flight and were predicted adequately by the AAW model. Overall, the aerodynamic model created for the AAW program proved to be adequate and was an improvement over the existing models for the particular vehicle used.

\section{References}

${ }^{1}$ Voracek, D., Pendleton, E., Reichenbach, E., Griffin, Dr. K., and Welch, L., "The Active Aeroelastic Wing Phase I Flight Research Through January 2003," NASA/TM-2003-210741, 2003.

${ }^{2}$ Dibley, R., Allen, M., Clarke, R., Gera, J., and Hodgkinson, J., "Development and Testing of Control Laws for the Active Aeroelastic Wing Program," AIAA-2005-6314 (to be published), 2005.

${ }^{3}$ Murray, J. E., and Maine, R. E., “pEst Version 2.1 User’s Manual,” NASA Technical Memorandum 88280, 1987.

${ }^{4}$ Maine, R. E., and Iliff, K. W., "Application of Parameter Estimation to Aircraft Stability and Control: the Output-Error Approach," NASA Reference Publication 1168, 1986.

${ }^{5}$ Moes, T. R., Noffz, G. K., and Iliff, K. W., "Results From F-18B Stability and Control Parameter Estimation Flight Tests at High Dynamic Pressures,” NASA/TP-2000-209033, 2003. 